\title{
Modeling and optimal control of dengue disease with screening and information
}

\author{
Akhil Kumar Srivastav $^{a, b *}$ Anuj Kumar ${ }^{c \dagger}$ Prashant K Srivastava $^{d \ddagger}$ \\ Mini Ghosh ${ }^{a \S}$ \\ ${ }^{a}$ Division of Mathematics, School of Advanced Sciences, \\ Vellore Institute of Technology, Chennai, India \\ ${ }^{b}$ Present Address: Basque Center for Applied Mathematics (BCAM), Bilbao, Spain, \\ ${ }^{c}$ School of Mathematics, Thapar Institute of Engineering and Technology, Patiala 147004, India \\ ${ }^{d}$ Department of Mathematics, Indian Institute of Technology Patna, Patna 801103, India
}

\begin{abstract}
This study presents a mathematical model for dengue transmission which quantifies two very important aspects: one- the impact of information based behavioural response and the other - the segregation of infected human population into two subclasses, detected and undetected. For the proposed model, the sensitivity analysis is conducted to identify the key model parameters which not only influence the basic reproduction number but also regulate the transmission of dengue. Further, in order to find the optimal pathways for suitable control interventions that reduce the dengue prevalence and economic burden, an optimal control problem is proposed by considering information-induced behavioural change, quarantine, screening, use of repulsive measures and culling of mosquitoes as control interventions. A weighted sum of various costs incurred in applied controls and the cost due to dengue disease (productivity loss) is incorporated in the proposed cost functional. The analysis of control system using Pontryagins Maximum Principle leads the existence of the optimal control profiles. Further, an exhaustive comparative study for seven different control strategies is conducted numerically. Our findings ensure that every individual control strategy has their own impact on reducing the cumulative count of infection as well as cost. The combined impact of all control interventions is highly effective and economically viable in controlling the prevalence of dengue. We also investigated the effect of the basic reproduction number on the designed control strategies and observed that the comprehensive use of controls keeps a strong tab on the infective even if the severity of epidemic is high.
\end{abstract}

Keywords: Dengue model; Information; Screening; Detection; Treatment; Sensitivity analysis; Optimal control.

\footnotetext{
*E-mail: akhilkumar.srivastav2016@vitstudent.ac.in, asrivastav@bcamath.org

${ }^{\dagger}$ E-mail:anujdubey17@gmail.com

${ }^{\ddagger}$ E-mail: pksri@iitp.ac.in

${ }^{\S}$ Corresponding Author,E-mail: minighosh@vit.ac.in
} 


\section{Introduction}

Dengue fever is a vector-borne disease. It is transmitted via the bite of female Aedes aegypti mosquitoes and is caused by four closely related dengue serotypes (DENV 1-4) [1,2]. Dengue affects almost all age groups. The symptoms of disease become apparent after 3-14 days of the bite of an infected mosquito. In recent years, the dengue cases are increasing globally and as per data from the World Health Organization there are 284528 million cases per year around the world [4]. Although no effective vaccine is available against dengue fever [5] at the moment but some clinical trials are in various phases. Thus, to avoid dengue infection healthy individuals have to prevent themselves from the mosquitoes. For this purpose, the use of insecticide or repellent and making people informed about using mosquito net and other protective measures are some useful ways to control the disease $[6,7,8,9]$. The proper and efficient diagnosis of dengue is very important in order to provide clinical care (which includes early detection of severe cases, confirmed cases etc), surveillance and control of dengue epidemic. It is also important for studying pathogenesis, extracting further knowledge and development of control measures such as vaccine. The diagnosis of dengue may involve detection of viral load, viral nucleic acid, antigens or antibodies or even a combination of all these. Normally, the virus is detected in bodily fluid and other tissues in about 5 days after onset of illness. [10].

With the support from WHO, the National Strategic Plan (NSP) for dengue disease control was established by the NVBDCP, Ministry of Health and Family Welfare, Government of India. Among the control strategies the factors include - EDPT (early case identification and prompt treatment), vector control, personal protection against mosquito bites, environmental management, and community awareness [11, 12]. The EDPT is considered to be basic technique for dengue control. All cases of dengue require drastic treatment to avoid dengue spread in the community. The control of mosquitoes in the community is possible using chemical and biological control. Mosquito repellent creams, liquids, coils, mats, and house screening with nets, use of insecticide-treated bed nets, and wearing clothing that covers the body's maximal surface area can all be utilized for personal protection. The identification and clearing the breeding sites, environmental management and community awareness, can also be effective strategies in mosquito control. In India, the Swachh Bharat Mission encourages people to keep their surroundings clean, eventually removing mosquito breeding grounds.

Many mathematical models have been proposed literature to study the various aspects of dengue transmission dynamics. In [14, 15] Esteva and Vargas proposed SIR-SI models taking constant and variable human populations respectively. They found the existence of the various equilibrium points and discussed their stability properties. In [16] Amaku et al. considered the impact of vector-control strategies on the human prevalence of dengue virus. They developed a mathematical model and discussed the sensitivity analysis of $\mathcal{R}_{0}$. Based on the sensitivity analysis they suggested that the control of adult mosquitoes can reduce the transmission of the dengue. In [17], Srivastav and Ghosh developed and analyzed a mathematical model with treatment by considering logistic growth of mosquito. They consiered the treatment rate proportional to the infective below the threshold for treatment and as constant for exceeding the threshold. They also performed parameter estimation with help of real data.

In [18], authors proposed a mathematical model of dengue disease by considering both 
mosquito and human population. They discussed vaccination and control strategies for the model. They have focused on two types of controls: mechanical control, to eliminate the mosquito breeding grounds, and chemical controls, to kill the dengue vector by using insecticides and larvicides. Abdelrazec et al. [19] proposed a mathematical model for the dengue fever where they considered nonlinear recovery rate and investigated the impact of available resources on the spread and control of the dengue disease.

Recently Srivastav et al. [20] studied impact of the early case detection and analysed it in both deterministic and stochastic setup. The authors divided infected population into undetected and detected which was further divided into moderately and severally infected population and results recommend that by sufficient management of quarantine/hospitalization, the disease prevalence may be reduced. Another study by Ghosh et al. indicates that the health-care organizations must focus on active case finding of symptomatic as well as asymptomatic individuals along with personal protection and mosquitoes control to achieve rapid reduction of dengue cases [21].

Optimal control is a mathematical tool that has been used in epidemiological models to explore the impact of various control interventions and also to provide relevant cost analysis. It provides helpful information in determining suitable control policy among the available control interventions and their combinations [28, 29]. Optimal control has also been used in dengue modeling to assess the impact of certain interventions. For example, Zheng and Nei discussed two strain dengue model in the presence of awareness and vector control [22]. With the help of optimal control problem they suggested that mosquito control and awareness about the dengue in the human population can reduce the disease prevalence. Blayneh et al. discussed the optimal control problem for vector-borne diseases. In this work they used transmission of the disease and treatment of the infective as control and suggested that if both controls are applied simultaneously, then disease control will be easy [23]. In [24] Rawson et al. showed that combination of sterile insect techniques for the mosquito population and vaccination for the human population is beneficial to reduce the disease in population. Tang et al. considered vaccination with insecticide administration and isolation with insecticide administration as two control policies for control of the dengue disease [25]. Rodrigues et al. studied an optimal control model using vaccination as control [26]. With the help of optimal control a costefficient strategy is investigated in [27]. They considered vaccination, applying insecticides to adult and aquatic mosquitoes, and reducing the number of manmade breeding places for the mosquitoes as controls.

Information about disease prevalence also plays a crucial role in the dynamics of the disease. This is because in presence of information, healthy individuals take precautionary and protective measures. Hence, this information induces behavioural change in the healthy population and they become less susceptible to the disease. This intervention, which is categorised as non-pharmaceutical interventions, is useful and can prove to be very effective in combination with other control interventions [29].

In this paper, we have proposed a mathematical model for dengue where we have considered the infected individuals being divided into two subclasses, undetected and detected. It is assumed that infection is spread via these and the interaction of host and vector is of standard mass action type. Further, we have also incorporated the idea of information induced behavioural change and consider such population into a separate compartment. As the informed individuals are using self protective measures, their infection probability is less. The 
model is then modified to an optimal control problem by choosing the information induced behavioural change, use of inhibitory measures to reduce infection, screening, detection and mosquito culling as control measures. Our aim in this paper is to find suitable control policy by finding an optimal combination of interventions so as to not only reduce the dengue load but also reduce the economic burden.

This paper is organized as follows: first, in section 2 we describe a mathematical model. In section 3 the model analysis is performed and some basic results are established. Section 4 deals with sensitivity analysis. In section 5 the optimal control problem is proposed and analysed which is followed by description of proposed policies. Exhaustive numerical simulation of the control measures is provided in section 6 before finally concluding the paper in section 7 .

\section{Mathematical Model}

In this section we propose a dengue spread compartmental model. We divide the total human population $N(t)$ into seven disjoint compartments, namely, susceptible individuals $(S)$, aware individuals $(A)$ who have information about the disease prevalence and hence are using protective measures, exposed individuals $(E)$ and infected individuals with dengue. The infected individuals are divided into two sub classes, the one which are detected for dengue as detected $\left(I_{1}\right)$, and and other undetected $\left(I_{2}\right)$ who posses dengue but are not detected so far. Furthermore compartments are of quarantine individuals $(Q)$ and recovered individuals from Dengue $(R)$. In addition we also consider the density of information as $(Z)$ along with its growth equation depending upon the total infective individuals [29], this information is assumed to be related with density of infective population (disease prevalence). When people come in contact with the information, they start taking protective measures as they become aware about the fatalities of the disease.Further, we choose the vector population, which is divided into two compartments, namely, susceptible mosquito population $\left(S_{v}\right)$ and infected mosquito population $\left(I_{V}\right)$.

The human population are recruited in the region at a constant rate $\Lambda$ (by birth or immigration) and are assumed to join the susceptible class. Individuals in susceptible class move the exposed class on effective contact with infected mosquitoes. The interactions between susceptible humans and infected mosquitoes are assumed to be of standard mass action type. Also susceptible human join aware class by getting informed. The rate of susceptible human and information is taken as $\eta$. Aware individuals join the exposed class on effective contact with infected mosquitoes, however the susceptibility of aware humans is lesser than susceptible humans because these individuals use the information of disease to protect themselves by using protective measures. The interactions between aware humans and infected mosquitoes are assumed to be of standard incidence type. The exposed human after latency become infected at a rate $\alpha$, where a fraction $m$ of these move to $I_{2}$ class and remaining $(1-m)$ are assumed to join $I_{1}$ class. After detection, from undetected infected population $I_{2}$ will join detected infected population with the rate of $\delta$. Detected infected human population are assumed to join quarantine/ hospitalized class $Q$ with the rate of $\gamma_{1}$, and after recovery quarantine/hospitalized population will move to recover class $R$ with the rate of $\alpha_{1}$. The information $(\mathrm{Z})$ is assumed to grow proportional to detected infective with rate $a I_{1}$ and it will degrade with time at rate $a_{0} Z$. 


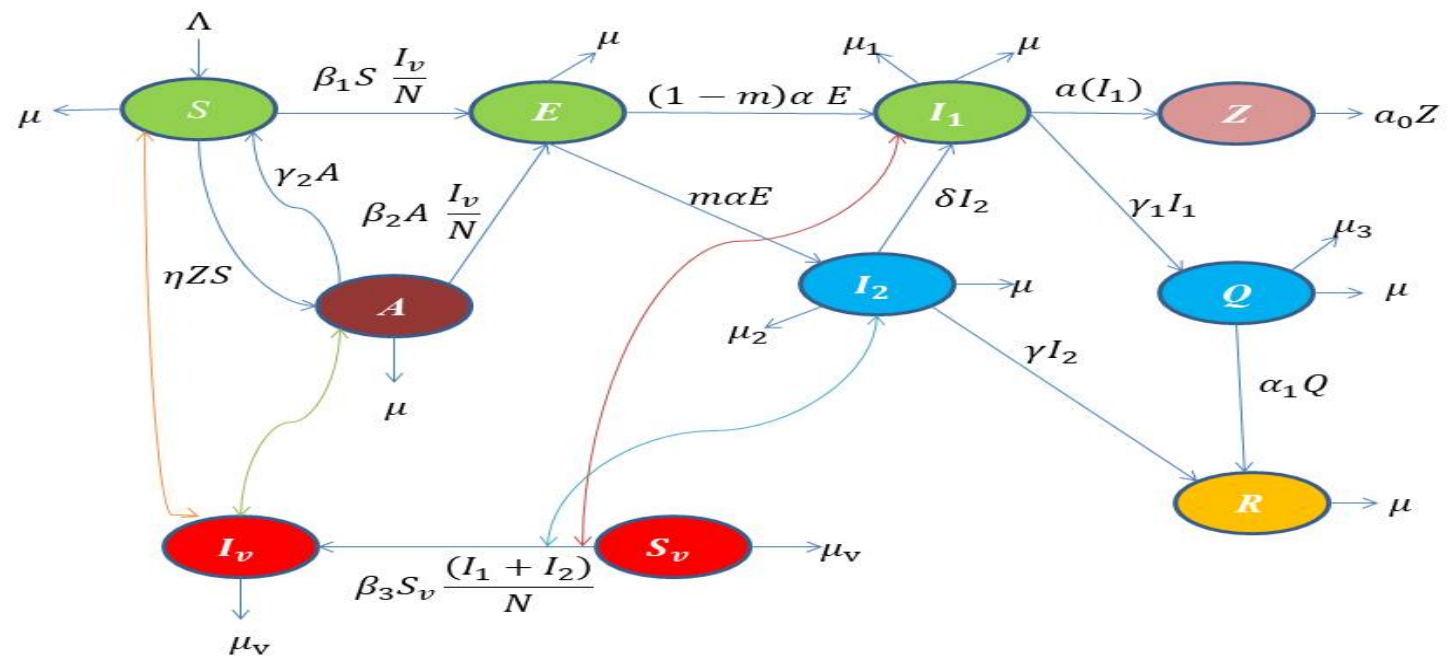

Figure 1: Flow diagram of the model.

The flow diagram is given in Figure-1 and model equations are as follows:

$$
\begin{aligned}
\frac{d S}{d t} & =\Lambda-\beta_{1} S \frac{I_{v}}{N}-\mu S-\eta Z S+\gamma_{2} A \\
\frac{d A}{d t} & =\eta Z S-\beta_{2} A \frac{I_{v}}{N}-\gamma_{2} A-\mu A \\
\frac{d E}{d t} & =\beta_{1} S \frac{I_{v}}{N}+\beta_{2} A \frac{I_{v}}{N}-\alpha E-\mu E \\
\frac{d I_{1}}{d t} & =(1-m) \alpha E-\left(\mu+\mu_{1}\right) I_{1}-\gamma_{1} I_{1}+\delta I_{2} \\
\frac{d I_{2}}{d t} & =m \alpha E-\left(\mu+\mu_{2}\right) I_{2}-\gamma I_{2}-\delta I_{2}, \\
\frac{d Q}{d t} & =\gamma_{1} I_{1}-\left(\mu+\alpha_{1}+\mu_{3}\right) Q \\
\frac{d R}{d t} & =\alpha_{1} Q+\gamma I_{2}-\mu R, \\
\frac{d Z}{d t} & =a I_{1}-a_{0} Z \\
\frac{d S_{v}}{d t} & =\Lambda_{v}-\beta_{3} S_{v}\left(\frac{I_{1}+I_{2}}{N}\right)-\mu_{v} S_{v}, \\
\frac{d I_{v}}{d t} & =\beta_{3} S_{v}\left(\frac{I_{1}+I_{2}}{N}\right)-\mu_{v} I_{v},
\end{aligned}
$$

where $\beta_{1}>\beta_{2}$ and $\mu_{2}>\mu_{1}$ and $N=S+A+E+I_{1}+I_{2}+Q+R, N_{v}=S_{v}+I_{v}$. 
Table 1: Description of parameters

\begin{tabular}{|c|c|c|c|c|}
\hline Parameter & & Description & Value & Reference \\
\hline (1) & : & Rate of recruitment in human population, & Varies & Demographic \\
\hline$\Lambda_{v}$ & : & Rate of recruitment in mosquito population, & Varies & Assumed \\
\hline$\beta_{1}$ & : & Transmission rate from $S$ to $I_{v}$ & 0.375 & {$[18]$} \\
\hline$\beta_{2}$ & : & Transmission rate from $A$ to $I_{v}$ & 0.2 & Assumed \\
\hline$\beta_{3}$ & : & Transmission rate from $S_{v}$ to infected class $\left(I_{1}+I_{2}\right)$, & 0.75 & {$[18]$} \\
\hline$\mu$ & : & Natural death rate for human population, & 0.0000425 & {$[37]$} \\
\hline$\mu_{1}$ & : & Disease related death rate for Detected human population, & 0.0004 & Assumed \\
\hline$\mu_{2}$ & : & Disease related death rate for undetected human population, & 0.004 & {$[20]$} \\
\hline$\gamma$ & : & recovery rate for undetected infected population, & 0.143 & {$[18]$} \\
\hline$\gamma_{1}$ & : & Progression rate from $I_{1}$ to $Q$ & 0.01 & Assumed \\
\hline$\gamma_{2}$ & : & Progression rate from $A$ to $S$, & 0.02 & {$[20]$} \\
\hline$\delta$ & : & Progression rate from $I_{2}$ to $I_{1}$, & 0.01 & Assumed \\
\hline$\alpha_{1}$ & : & Progression rate from $Q$ to $R$ & 0.143 & {$[18]$} \\
\hline$\mu_{v}$ & : & Natural death of the mosquito population. & 0.042 & {$[18]$} \\
\hline$\eta$ & : & Awareness of susceptible human population rate & 0.1 & {$[28]$} \\
\hline$m$ & : & Fraction of Exposed human population rate & 0.2 & Assumed \\
\hline$\alpha$ & : & Progression rate from $E$ to $I_{1}$ and $I_{2}$ & 0.1 & [18] \\
\hline$a_{0}$ & : & Degradation rate of Information & 0.05 & [28] \\
\hline$\mu_{3}$ & : & Disease related death rate for Quarantine human population, & 0.004 & Assumed \\
\hline
\end{tabular}




\subsection{Positivity and boundedness of the solutions}

Here, we shall show the positivity and boundedness of all populations. From the model system (1), we have

$$
\begin{gathered}
\left.\frac{d S}{d t}\right|_{S=0}=\Lambda+\gamma_{2} A>0,\left.\quad \frac{d A}{d t}\right|_{A=0}=\eta Z S>\left.0 \quad \frac{d E}{d t}\right|_{E=0}=\beta_{1} S \frac{I_{v}}{N}+\beta_{2} A \frac{I_{v}}{N} \geq 0 \\
\left.\frac{d I_{1}}{d t}\right|_{I_{1}=0}=(1-m) \alpha E+\delta I_{2} \geq 0,\left.\quad \frac{d I_{2}}{d t}\right|_{I_{2}=0}=m \alpha E \geq 0,\left.\quad \frac{d Q}{d t}\right|_{Q=0}=\gamma_{1} I_{1} \geq 0 \\
\left.\frac{d R}{d t}\right|_{R=0}=\alpha_{1} Q+\gamma I_{2} \geq 0,\left.\quad \frac{d Z}{d t}\right|_{Z=0}=a I_{1} \geq 0,\left.\quad \frac{d S_{v}}{d t}\right|_{S_{v}=0}=\Lambda_{v}>0, \\
\left.\frac{d I_{v}}{d t}\right|_{I_{v}=0}=\beta_{3} S_{v}\left(\frac{I_{1}+I_{2}}{N}\right) \geq 0 .
\end{gathered}
$$

Here all the rates are non-negative, so if we start in the interior of the non-negative bounding cone $\mathbb{R}^{10}$, we shall always remain in this cone keeping in view the fact that the direction of the vector field is inward on all the bounding planes. Thus non-negativity of all the solutions of the model system (1) is guaranteed.

Further from the model system (1), we note that the total population $N=S+A+E+$ $I_{1}+I_{2}+Q+R$, and $N_{v}=S_{v}+I_{v}$ follows the following differential equation

$$
\frac{d N}{d t}=\Lambda-\mu N-\mu_{1} I_{1}-\mu_{2} I_{2}-\mu_{3} Q
$$

and

$$
\frac{d N_{v}}{d t}=\Lambda_{v}-\mu_{v} N_{v}
$$

This gives $\limsup _{t \rightarrow \infty} N \leq \frac{\Lambda}{\mu}, \limsup _{t \rightarrow \infty} N_{v} \leq \frac{\Lambda_{v}}{\mu_{v}}$. Therefore all the solutions $S(t), A(t), E(t)$, $I_{1}(t), I_{2}(t), Q(t)$ and $R(t)$ are bounded by $\frac{\Lambda}{\mu}$ and the solutions $S_{v}(t)$ and $I_{v}(t)$ are bounded above by $\frac{\Lambda_{v}}{\mu_{v}}$. Further, $Z(t)$ is bounded by $\frac{\Lambda a}{\mu a_{0}}$. Hence the biologically feasible region of the model system (1) is given by the following positively invariant set:

$\Omega=\left\{\left(S, A, E, I_{1}, I_{2}, Q, R, Z, S_{v}, I_{v}\right) \in \mathbb{R}^{10}: 0 \leq S, A, E, I_{1}, I_{2}, Q, R \leq \frac{\Lambda}{\mu}, 0 \leq S_{v}, I_{v} \leq \frac{\Lambda_{v}}{\mu_{v}}\right.$ and $\left.0 \leq Z \leq \frac{\Lambda a}{\mu a_{0}}\right\}$.

\section{Existence of Equilibrium Points and the Basic Reproduc- tion Number}

\subsection{Disease-free equilibrium $E_{0}$}

We consider the system (1) and find the disease-free equilibrium. For our model we have disease-free equilibrium as

$$
E_{0}:=\left(N^{0}=\frac{\Lambda}{\mu}, 0,0,0,0,0,0,0, N_{v}^{0}=\frac{\Lambda_{v}}{\mu_{v}}, 0\right)
$$




\subsection{The basic reproduction number}

We find the basic reproduction number $\left(\mathcal{R}_{0}\right)$ by following the next generation matrix method [32] we find the matrix $\mathcal{F}$ and $\mathcal{V}$ as follows:

$$
\mathcal{F}=\left(\begin{array}{c}
\beta_{1} S \frac{I_{v}}{N}+\beta_{2} A \frac{I_{v}}{N} \\
0 \\
0 \\
\beta_{3} S_{v} \frac{\left(I_{1}+I_{2}\right)}{N}
\end{array}\right) \text { and } \mathcal{V}=\left(\begin{array}{c}
(\alpha+\mu) E \\
-(1-m) \alpha E+\left(\mu+\mu_{1}+\gamma_{1}\right) I_{1}-\delta I_{2} \\
-m \alpha E+\left(\mu+\mu_{2}+\gamma+\delta\right) I_{2} \\
\mu_{v} I_{v}
\end{array}\right)
$$

Following [32] we calculate

$\mathrm{F}=$ Jacobian of $\mathcal{F}$ at $E_{0}=\left(\begin{array}{cccc}0 & 0 & 0 & \beta_{1} \\ 0 & 0 & 0 & 0 \\ 0 & 0 & 0 & 0 \\ 0 & \beta_{3} \frac{N_{v}^{0}}{N^{0}} & \beta_{3} \frac{N_{v}^{0}}{N^{0}} & 0\end{array}\right)$

and $\mathrm{V}=$ Jacobian of $\mathcal{V}$ at $E_{0}=\left(\begin{array}{cccc}\alpha+\mu & 0 & 0 & 0 \\ -(1-m) \alpha & \left(\mu+\mu_{1}+\gamma_{1}\right) & -\delta & 0 \\ -m \alpha & 0 & \left(\mu+\mu_{2}+\gamma+\delta\right) & 0 \\ 0 & 0 & 0 & \mu_{v}\end{array}\right)$,

and find that

$$
F V^{-1}=\left(\begin{array}{cccc}
0 & 0 & 0 & \frac{\beta_{1}}{\mu_{v}} \\
0 & 0 & 0 & 0 \\
0 & 0 & 0 & 0 \\
a_{41} & a_{42} & a_{43} & 0
\end{array}\right)
$$

Where

$$
\begin{gathered}
a_{41}=\frac{\beta_{3} \alpha N_{v}^{0}\left[m\left(\gamma_{1}+\mu+\mu_{1}\right)+\delta+(1-m)\left(\gamma+\mu+\mu_{2}\right)\right]}{N^{0}(\alpha+\mu)\left(\mu+\mu_{2}+\gamma+\delta\right)\left(\gamma_{1}+\mu+\mu_{1}\right)}, \\
a_{42}=\frac{N_{v}^{0} \beta_{3}}{N^{0}\left(\gamma_{1}+\mu+\mu_{1}\right)}, a_{43}=\frac{N_{v}^{0} \beta_{3}\left(\gamma_{1}+\mu+\mu_{1}+\delta\right)}{N^{0}\left(\delta+\gamma+\mu+\mu_{2}\right)\left(\gamma_{1}+\mu+\mu_{1}\right)},
\end{gathered}
$$

The largest eigenvalue of $F V^{-1}$ is defined as the basic reproduction number $\mathcal{R}_{0}$ and is given as follows:

$$
\mathcal{R}_{0}=\sqrt{\frac{\beta_{1}}{\mu_{v}} a_{41}} .
$$

The quantity $\mathcal{R}_{0}$ is known as basic reproduction number, the expected number of secondary cases produced in completely susceptible population, by a typical infective individual. where the term $\frac{\beta_{1}}{\mu_{v}}$ denotes the expected number of human generated by single infected mosquito, and the term

$$
\frac{\beta_{3} \alpha N_{v}^{0}\left[m\left(\gamma_{1}+\mu+\mu_{1}\right)+\delta+(1-m)\left(\gamma+\mu+\mu_{2}\right)\right]}{N^{0}(\alpha+\mu)\left(\mu+\mu_{2}+\gamma+\delta\right)\left(\gamma_{1}+\mu+\mu_{1}\right)},
$$


denotes the expected number of infected mosquitoes generated by single infected human.

\section{Numerical Simulation}

\subsection{Forward sensitivity analysis and impact of parameters on the basic reproduction number}

We establish the normalized forward sensitivity indices of the basic reproduction number with respect to model parameters [21]. For that we use the forward sensitivity index of a variable, with respect to a given parameter, which is defined as the ratio of the relative change in the variable to the relative change in the parameter. If such variable is differentiable with respect to the parameter, then the sensitivity index is defined using partial derivatives. The forward sensitivity index of $\mathcal{R}_{0}$, which is differentiable with respect to a given parameter $\mathrm{p}$, is defined by

$$
\gamma_{p}^{\mathcal{R}_{0}}=\frac{\partial \mathcal{R}_{0}}{\partial p} \frac{p}{\mathcal{R}_{0}}
$$

One can easily compute an analytical expression for the sensitivity of $\mathcal{R}_{0}$, using the above formula, to each parameter that it includes. In Fig. 2, we plot the sensitivity indices of $R_{0}$ with respect to the parameters of interest. Evidently, Fig. 5 suggests that the magnitude of $\mathcal{R}_{0}$ increases with increase in the values of parameters $\beta_{1}, \beta_{3}, \mu$ and $\Lambda_{v}$ as these parameters possess positive indices with $\mathcal{R}_{0}$. Similarly, the parameters having negative indices with $\mathcal{R}_{0}$ are $m, \gamma_{1}, \gamma, \mu_{1}, \mu_{2}, \mu_{v}$ and $\Lambda$. Hence, increments in these parameters cause decline in the value of $\mathcal{R}_{0}$. It is clear that occurrence of a lower value of $\mathcal{R}_{0}$ helps to prevent the disease prevalence. Thus, to wipe out the disease from the system, we must control the increase of the parameters having positive indices with basic reproduction number whereas parameters which have negative indices should be sustained. Therefore, any prevention measure which can reduce the disease burden must be seriously considered by the health-care ocials to control the subsequent outbreaks.

We have plotted contour plot of some key parameters on $\mathcal{R}_{0}$. It shows that in plot (3a) $\beta_{1}$ and $\beta_{3}$ has same effect on $\mathcal{R}_{0}$, which means if we increase or decrease the value of $\beta_{1}$ and $\beta_{3}$, then the value of $\mathcal{R}_{0}$ will increase or decrease respectively, from the $(3 \mathrm{~b})$ we can conclude that increase the value of $\Lambda_{v}$ will increase the value of the $\mathcal{R}_{0}$ but if we increase the value of $\mu_{v}$, it will decrease the value of decrease the value of the $\mathcal{R}_{0}$, which is the death rate of the mosquito and this is the one of the best control policy to reduce the disease infection from the population. Similarly from the figure $(3 \mathrm{c}, 3 \mathrm{~d})$, increase in the value of the $\beta_{1}$ and $\delta$ will increase the value of the $\mathcal{R}_{0}$ and increase the value of $\gamma_{1}$ and $\delta$ will decrease the value of $\mathcal{R}_{0}$.

\subsection{Sensitivity Analysis}

We employed a global sensitivity analysis to assess the impact of uncertainty and the sensitivity of the outcomes of the numerical simulations to variations in each parameter of the model using Latin Hypercube Sampling (LHS) and partial rank correlation coefficients (PRCC) $[33,34]$. To generate the LHS matrices, we assume that all the model parameters 


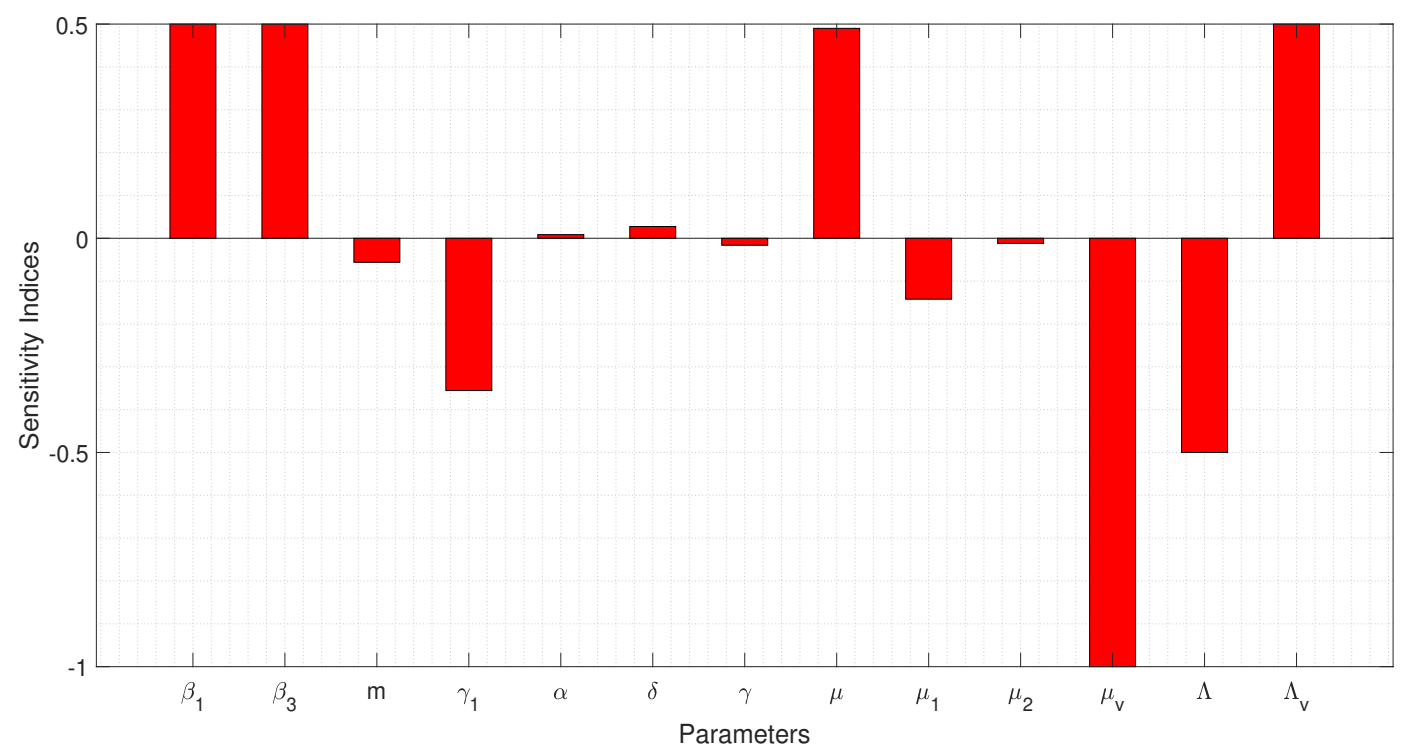

Figure 2: Normalized forward sensitivity indices of $\mathcal{R}_{0}$ with respect to model parameters. Parameter values: $\Lambda=200, \quad \Lambda_{v}=1000, \quad \beta_{1}=0.375, \quad \beta_{3}=0.75, \quad \mu=0.000042, \quad \mu_{2}=$ $0.0004, \quad \mu_{2}=0.004, \quad \gamma=0.143, \quad \gamma_{1}=0.01, \quad \alpha=0.1, \quad \delta=0.01, \quad m=0.2$

are uniformly distributed. We calculate partial rank correlation coefficients (PRCCs) between the parameters $\Lambda, \Lambda_{v}, \beta_{1}, \beta_{2}, \beta_{3}, \mu, \mu_{1}, \mu_{2}, \gamma, \gamma_{1}, \gamma_{2}, \delta, \alpha_{1}$ and $\mu_{v}$ from system (1) with Exposed Human Population(E), Infected and Detected human populations $\left(I_{1}\right)$, Infected and undetected human populations $\left(I_{2}\right)$ and Infected mosquitoes population $\left(I_{v}\right)$. Nonlinear and monotone relationships are observed for Exposed Human Population(E), Infected and Detected human populations $\left(I_{1}\right)$, Infected and undetected human populations $\left(I_{2}\right)$ and Infected mosquitoes population $\left(I_{v}\right)$ with the input parameters of the model, which is a prerequisite for computing PRCCs. Then, simulations of the model per LHS run were carried out, using the baseline values tabulated in Table 2 and the ranges as $25 \%$ from the baseline values (in either direction). The indexes are evaluated at the time points 1000 days and the bar diagram of the PRCC values are shown in Figs. 4(a), 4(b), 4(c) and 4(d) for Exposed Human Population(E), Infected and Detected human populations $\left(I_{1}\right)$, Infected and undetected human populations $\left(I_{2}\right)$ and Infected mosquitoes population $\left(I_{v}\right)$ respectively. Based on the sensitivity analysis results, we are formulating control model.The details description is provided in following section.

\section{Optimal Control Problem}

In this section, we extend the mathematical model (1) to propose a corresponding optimal control problem by introducing various control interventions to minimise the dengue burden as well as financial loss generated. Here, we quantify five different types of control interventions, namely, $u_{1}(t), u_{2}(t), u_{3}(t), u_{4}(t), u_{5}(t)$, and these are either pharmaceutical or 


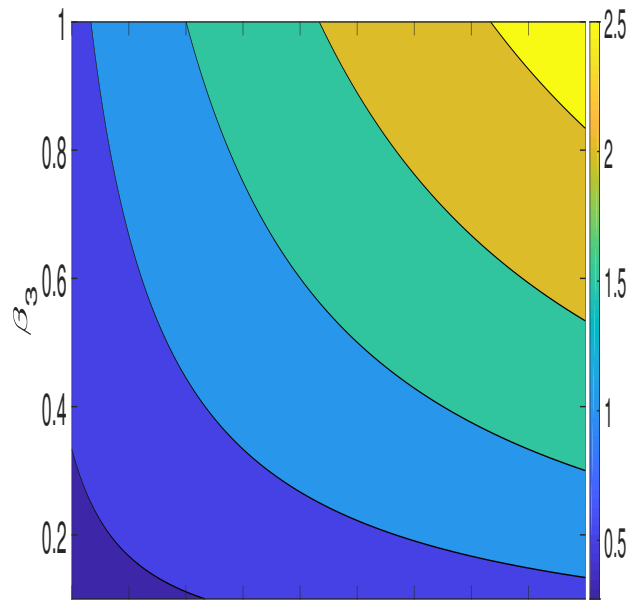

$\begin{array}{lllllllll}0.1 & 0.2 & 0.3 & 0.4 & 0.5 & 0.6 & 0.7 & 0.8 & 0.9\end{array}$

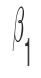

(a)

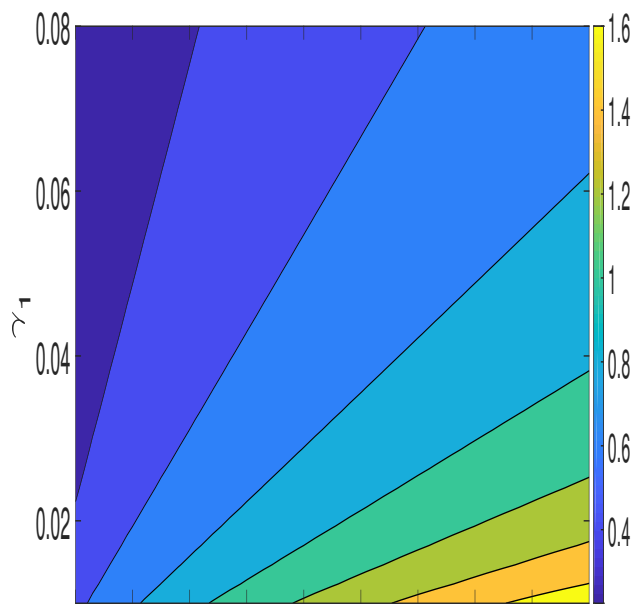

$\begin{array}{llllllllllll}0.1 & 0.2 & 0.3 & 0.4 & 0.5 & 0.6 & 0.7 & 0.8 & 0.9\end{array}$

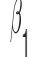

(c)

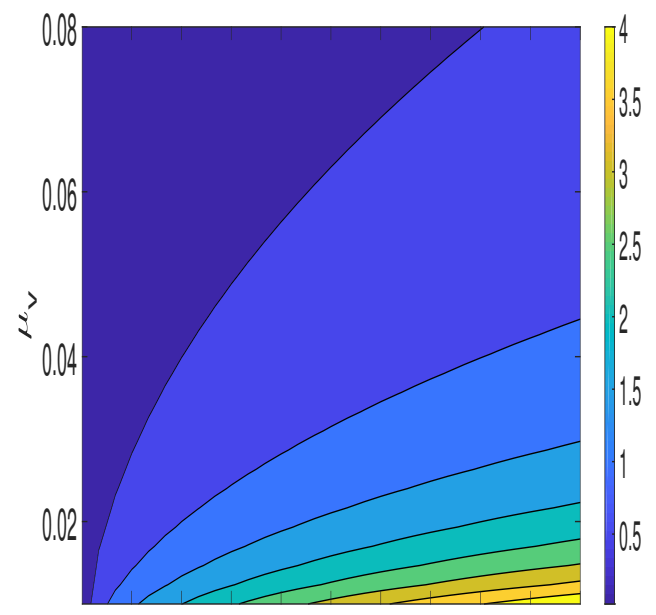

1002003004005006007008009001000

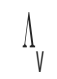

(b)

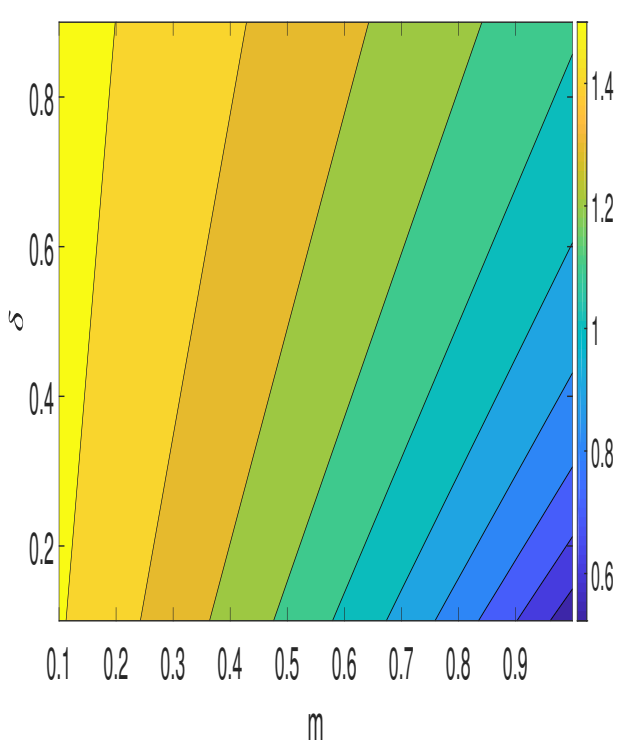

(d)

Figure 3: Contour plots of the basic reproduction number $\left(\mathcal{R}_{0}\right)$ with respect to (a) $\beta_{1}$ and $\beta_{3}$ (b) $\Lambda_{v}$ and $\mu_{v}$ (c) $\beta_{1}$ and $\gamma_{1}$ (d) $m$ and $\delta$ for the Parameter values: $\Lambda=200, \Lambda_{v}=$ $1000, \quad \beta_{1}=0.375, \quad \beta_{3}=0.75, \quad \mu=0.000042, \quad \mu_{2}=0.0004, \quad \mu_{2}=0.004, \quad \gamma=0.143, \quad \gamma_{1}=$ $0.01, \quad \alpha=0.143, \quad \delta=0.01$

non-pharmaceutical. The details of each intervention are described as follows: 


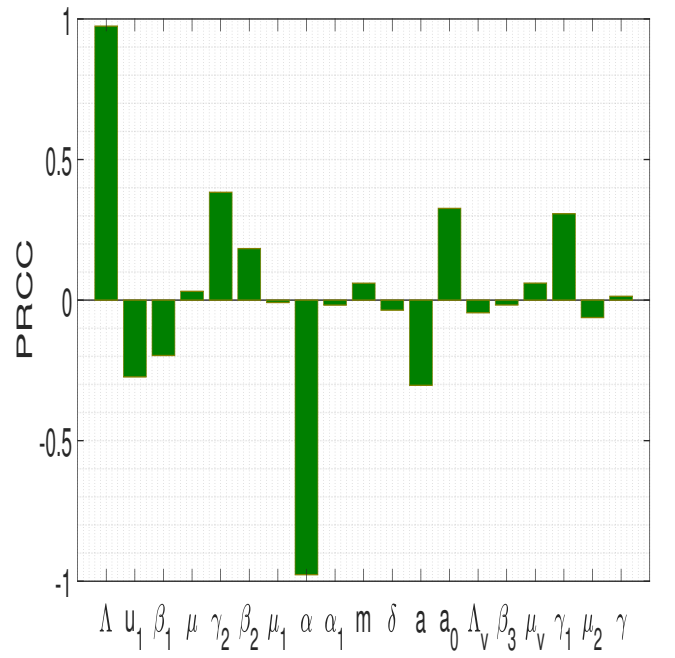

(a)

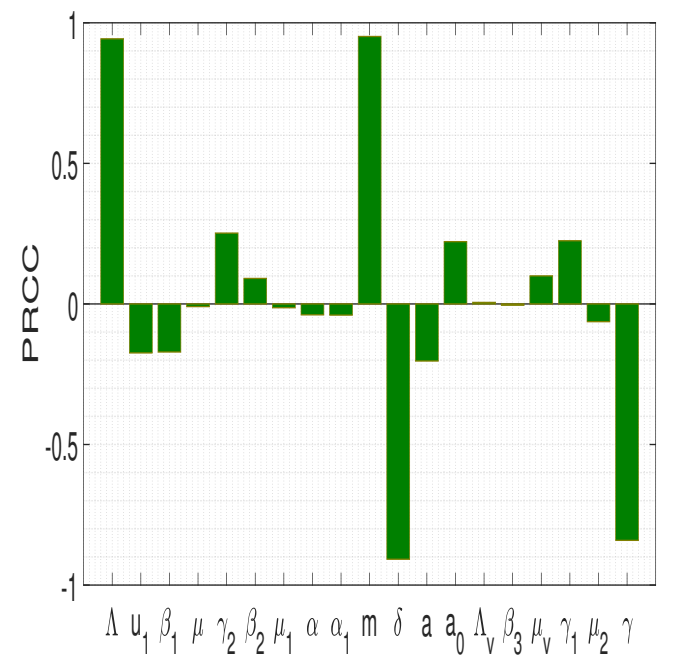

(c)

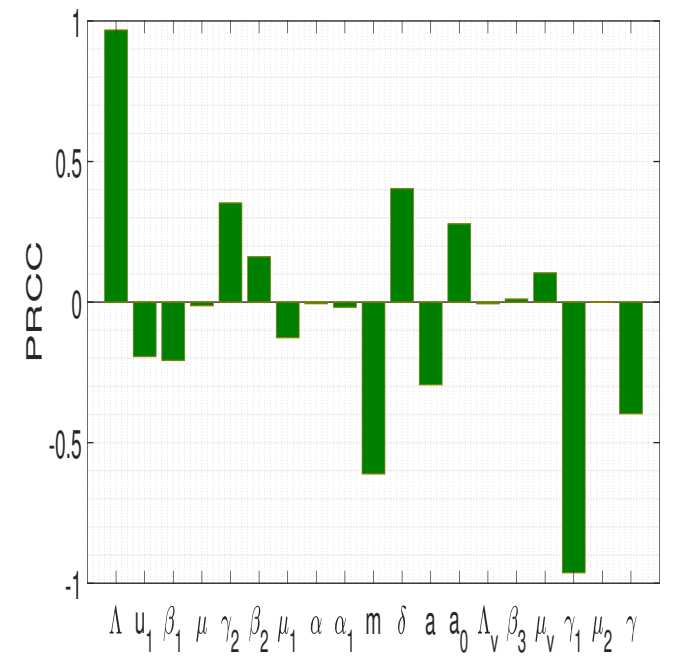

(b)

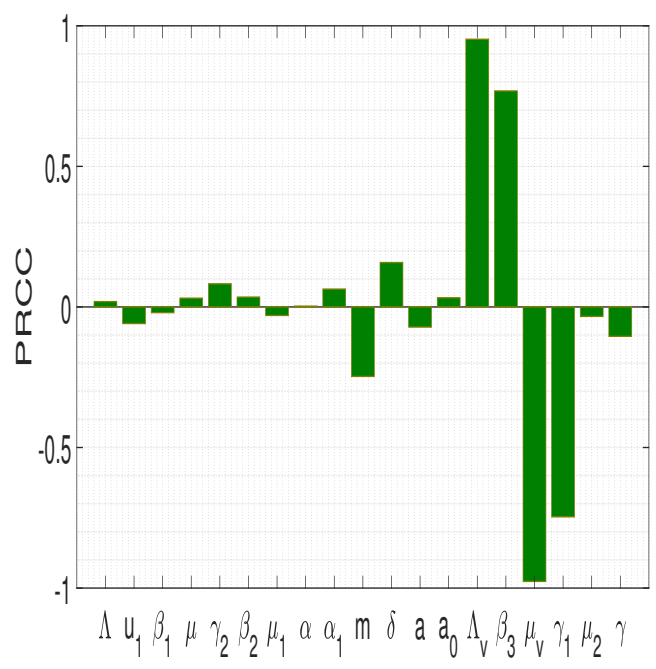

(d)

Figure 4: PRCC Plots (a) exposed human population (E), (b) infected and detected human populations $\left(I_{1}\right),(\mathrm{c})$ infected and undetected human populations $\left(I_{2}\right),(\mathrm{d})$ infected mosquitoes population $\left(I_{v}\right)$ 
- Control variable $u_{1}(t)$ : Due to the disease prevalence, the information for the same spreads within the population and induces the healthy individuals to take protective measures against the dengue disease. This rate for protective measure will depend on the severity of disease (density of infective population). Our aim is to optimize the use of information-induced protection and minimize the corresponding cost. Therefore, we introduce the intensity of behavioural response via information $u_{1}(t)$ as a variable control measure to protect the susceptible individuals so that maximum individuals get protected with minimum cost involved. The spread of information control considered here depends on limited resources that are put in through media, TV, newspaper and the protection coverage against the dengue disease. Therefore, due to the limitation of resources, we consider restriction on control which is defined below.

- Control variable $u_{2}(t)$ : This intervention represents the use of insecticide-treated bed nets, mosquito repulsive lotions and electronic devices to reduce transmission between mosquitoes and human. If one can reduce mosquitoes to human and human to mosquitoes transmission rate, then it will be helpful in controlling the mosquito-borne disease. Hence, here, we consider $u_{2}(t)$ as a control intervention to reduce humanmosquito interaction.

- Control variable $u_{3}(t)$ : The control variable $u_{3}(t)$ represents to send the detected infected human individuals into quarantine class for fast treatment and considered as quarantine control. As has been found that the quarantine is effective intervention and consumes huge amount of money. Thus the main objective is to minimise this population with minimum cost. we are using the term $\eta_{1} u_{3}(t)$ where $\eta_{1}$ is the additional quarantine rate of the detected infected individual undergoing quarantine for the better treatment $\left(\right.$ i.e. $\left(\gamma_{1}+\eta_{1} u_{3}(t)\right)=$ quarantine rate with treatment), is inserted in model.

- Control variable $u_{4}(t)$ : Since, the undetected infected are very harmful from the social well-being point of view. Therefore, the detection of such individuals becomes an important task. Thus, the control $u_{4}(t)$ represents to improve screening and detection of the undetected infected human population and quantified as a screening control. Similarly the term $\eta_{2} u_{4}(t)$ where $\eta_{2}$ is the additional detection rate for the fast screening and detection of the undetected infected individual will join detected infected individuals (i.e. $\left(\delta+\eta_{2} u_{4}(t)\right)=$ detection rate), is inserted in model.

- Control variable $u_{5}(t)$ : It represents the time dependent additional deaths of mosquitoes due to insecticide treatment. This is very important strategy for mosquito control. But at the same, a large amount of money is involved in implementing of such kind of interventions. Thus, we seek to find the optimal way for this. the term $\eta_{3} u_{5}(t)$ where $\eta_{3}$ is the additional death rate for the control of Aedes mosquito $\left(i . e .\left(\mu_{v}+\eta_{3} u_{5}(t)\right)=\right.$ death rate of mosquito), is inserted in model.

Due to restriction on medical resources, it is important to impose some bounds on controls as $0 \leq u_{1}(t), u_{2}(t), u_{3}(t), u_{4}(t), u_{5}(t) \leq 1$. Here, if $u_{1}(t), u_{2}(t), u_{3}(t), u_{4}(t)$, and $u_{5}(t)$ are zero, then there is no effort being placed in these controls at time $t$ whereas one represents the maximum capacity of applied interventions. Keeping in view of the above assumptions, the optimal control model is formulated as follows: 


$$
\begin{aligned}
\frac{d S}{d t} & =\Lambda-\left(1-u_{2}(t)\right) \beta_{1} S \frac{I_{v}}{N}-\mu S-\left(\eta+\theta u_{1}(t)\right) Z S+\gamma_{2} A \\
\frac{d A}{d t} & =\left(\eta+\theta u_{1}(t)\right) Z S-\beta_{2} A \frac{I_{v}}{N}-\gamma_{2} A-\mu A \\
\frac{d E}{d t} & =\left(1-u_{2}(t)\right) \beta_{1} S \frac{I_{v}}{N}+\beta_{2} A \frac{I_{v}}{N}-\alpha E-\mu E \\
\frac{d I_{1}}{d t} & =(1-m) \alpha E-\left(\mu+\mu_{1}\right) I_{1}-\left(\gamma_{1}+\eta_{1} u_{3}(t)\right) I_{1}+\left(\delta+\eta_{2} u_{4}(t)\right) I_{2} \\
\frac{d I_{2}}{d t} & =m \alpha E-\left(\mu+\mu_{2}\right) I_{2}-\gamma I_{2}-\left(\delta+\eta_{2} u_{4}(t)\right) I_{2} \\
\frac{d Q}{d t} & =\left(\gamma_{1}+\eta_{1} u_{3}(t)\right) I_{1}-\left(\mu+\mu_{3}+\alpha_{1}\right) Q \\
\frac{d R}{d t} & =\alpha_{1} Q+\gamma I_{2}-\mu R \\
\frac{d Z}{d t} & =a I_{1}-a_{0} Z, \\
\frac{d S_{v}}{d t} & =\Lambda_{v}-\left(1-u_{2}(t)\right) \beta_{3} S_{v}\left(\frac{I_{1}+I_{2}}{N}\right)-\left(\mu_{v}+\eta_{3} u_{5}(t)\right) S_{v} \\
\frac{d I_{v}}{d t} & =\left(1-u_{2}(t)\right) \beta_{3} S_{v}\left(\frac{I_{1}+I_{2}}{N}\right)-\left(\mu_{v}+\eta_{3} u_{5}(t)\right) I_{v}
\end{aligned}
$$

For further analysis, we write $u_{1}(t)=u_{1}, u_{2}(t)=u_{2}, u_{3}(t)=u_{3}, u_{4}(t)=u_{4}$ and $u_{5}(t)=u_{5}$ for our convenience.

\subsection{Cost construction and characterization of optimal controls}

This particular section devotes into two parts which include determination of the total cost

generated due to applied controls as well as diseases itself. Whereas the second part determines the analytical forms of the controls.

\subsubsection{Total cost determination}

Here, the total cost is determined for the applied control interventions and disease as well, which need to be minimized.

- Cost due to disease: The cumulative cost incurred due to the disease burden is modeled as follows:

$$
\int_{0}^{T}\left(C_{1} E+C_{2} I_{1}+C_{3} I_{2}-C_{4} A+C_{5}\left(S_{v}+I_{v}\right) d t\right.
$$

This cost consists of various components such as cost due to loss of manpower, opportunity loss and other related wealth loss.

- Cost incurred in enhancing information-induced behavioural response: The cost generated in information-induced behavioural response is defined as

$$
\int_{0}^{T} D_{1} u_{1}^{4} d t
$$


The term $D_{1} u_{1}^{4}$ describes the temporal cost generated in inducing the behaviour of individuals via information which is governed by various awareness programs and educational activities during the course of epidemic. Therefore, the total cost incurred in process primarily includes the cost involved in awareness and educational campaigns, cost of required manpower etc. Thus, in this case, we consider a relatively high nonlinearity in cost as $\left(u_{1}^{4}(t)\right)$ to account for the higher steepness in cost increment to cover same fraction of effort at higher level.

- Cost incurred in insecticide-treated bed nets : The total cost involved in reducing the transmission between human to mosquito is given as

$$
\int_{0}^{T} D_{2} u_{2}^{2} d t .
$$

Insecticide-treated bed nets (ITNs) are a form of personal protection that has been shown to reduce mosquito borne disease i.e. dengue, malaria etc. and death due to mosquito borne disease in endemic regions. But at the same time, the execution of ITNs requires lot of money which is given by the term $D_{2} u_{2}^{2}$. Therefore, here, we have considered a standard second order nonlinearity in this cost component.

- Cost incurred in treatment for detected infected human population: The cumulative cost in process of treating infected human is defined by

$$
\int_{0}^{T} D_{3} u_{3}^{2} d t
$$

Once, an individual becomes infected, treatment is the only possible way to contain the disease and that process consumes significant amount of money. So, here, the term $D_{3} u_{3}^{2}$ describes the cost involved in treatment for detected infected human population which primarily consists of the cost generated due to medical facilities, Hospitals, manpower etc.

- Cost incurred in screening and detection in undetected human population: The cost generated due to screening and detection process for undetected population is modeled by

$$
\int_{0}^{T} D_{4} u_{4}^{2} d t
$$

The term $D_{4} u_{4}^{2}$ describes the cost involved in screening and detection in human population. The total cost incurred in this coverage includes the cost due to screening campaigns, cost of kits used for detection and cost of other relevant measures.

- Cost incurred due to execution of insecticide treatment measure for mosquitoes: The cumulative cost involved due to insecticides to enhance the additional deaths of mosquitoes is defined as

$$
\int_{0}^{T} D_{5} u_{5}^{4} d t
$$

The term $D_{5} u_{5}^{4}$ describes the temporal cost generated by use of insecticides for additional deaths of mosquitoes. In this case, the components of total cost mainly include 
the cost due to implementation of insecticide measures (insecticide machine, manpower etc.). Since, the execution of such intervention not only requires huge manpower but also imposes significantly higher economic burden. Therefore, here, we consider a relatively high nonlinearity in the cumulative cost as $\left(u_{5}^{4}(t)\right)$ to account for the higher steepness in cost increment to cover same fraction of effort at higher level.

The cost functional corresponding to total cost incurred, for fixed time $T$, which need to be minimised is given by

$J=\int_{0}^{T}\left(C_{1} E+C_{2} I_{1}+C_{3} I_{2}-C_{4} A+C_{5}\left(S_{v}+I_{v}\right)+D_{1} u_{1}^{4}+D_{2} u_{2}^{2}+D_{3} u_{3}^{2}+D_{4} u_{4}^{2}+D_{5} u_{5}^{4}\right) d t$.

subject to the model system (2).

Here, the parameter $C_{1}, C_{2}, C_{3}, C_{4}, C_{5}, D_{1}, D_{2}, D_{3}, D_{4}$, are $D_{5}$ positive weight constants that not only balance the unit of integrands but also measure the relative costs. Our objective is to find the controls $u_{1}{ }^{*}, u_{2}{ }^{*}, u_{3}{ }^{*}, u_{4}{ }^{*}$ and $u_{5}{ }^{*}$, such that

$$
J\left(u_{1}^{*}, u_{2}^{*}, u_{3}^{*}, u_{4}^{*}, u_{5}^{*}\right)=\min _{\left(u_{1}, u_{2}, u_{3}, u_{4}, u_{5}\right) \in U} J\left(u_{1}, u_{2}, u_{3}, u_{4}, u_{5}\right),
$$

where $U$ is the control set and is defined as

$$
U=\left\{u_{1}, u_{2}, u_{3}, u_{4}, u_{5}: 0 \leq u_{1}, u_{2}, u_{3}, u_{4}, u_{5} \leq 1, t \in[0, T]\right\} .
$$

Here, all the controls are bounded and measurable.

\subsubsection{Existence and characterization of optimal controls}

Here, we shall first establish the existence of such control functions that minimises the cost functional $J$. The Lagrangian of this problem is defined as:

$$
\begin{aligned}
L\left(S, A, E, I_{1}, I_{2}, Q, R, Z, S_{v}, I_{v}, u_{1}, u_{2}, u_{3}, u_{4}, u_{5}\right)= & C_{1} E+C_{2} I_{1}+C_{3} I_{2}-C_{4} A+C_{5}\left(S_{v}+I_{v}\right) \\
& +D_{1} u_{1}{ }^{4}+D_{2} u_{2}{ }^{2}+D_{3} u_{3}{ }^{2}+D_{4} u_{4}{ }^{2}+D_{5} u_{5}{ }^{4} .
\end{aligned}
$$

Theorem 5.1. There exist optimal controls $u_{1}{ }^{*}, u_{2}{ }^{*}, u_{3}{ }^{*}, u_{4}{ }^{*}, u_{5}{ }^{*} \in U$ such that

$$
J\left(u_{1}{ }^{*}, u_{2}{ }^{*}, u_{3}{ }^{*}, u_{4}{ }^{*}, u_{5}{ }^{*}\right)=\min J\left(u_{1}, u_{2}, u_{3}, u_{4}, u_{5}\right)
$$

subject to system (2).

Proof. To establish this result, we follow the Theorem 4.1 mentioned in [39] for the existence of optimal controls. As, we have discussed above that all the state variables (population) are bounded for each bounded controls coming from the control set $U$. Furthermore, Lipschitz condition with respect to state variables is satisfied by the right hand part of the model system (2). The control variable set $U$ is also convex and closed by the definition and the model system (2) is linear in control variables. The integrand of the functional $L=C_{1} E+C_{2} I_{1}+$ $C_{3} I_{2}-C_{4} A+C_{5}\left(S_{v}+I_{v}\right)+D_{1} u_{1}^{4}+D_{2} u_{2}^{2}+D_{3} u_{3}^{2}+D_{4} u_{4}^{2}+D_{5} u_{5}^{4}$ is convex on the control set $U$ due to biquadratic and quadratic nature of control variables. Moreover, $L=C_{1} E+C_{2} I_{1}+$ 
$C_{3} I_{2}-C_{4} A+C_{5}\left(S_{v}+I_{v}\right)+D_{1} u_{1}^{4}+D_{2} u_{2}^{2}+D_{3} u_{3}^{2}+D_{4} u_{4}^{2}+D_{5} u_{5}^{4} \geq D_{1} u_{1}^{4}+D_{2} u_{2}^{2}+D_{3} u_{3}^{2}+$ $D_{4} u_{4}{ }^{2}+D_{5} u_{5}{ }^{4}$. Now, consider $c_{1}=\min \left(D_{1}, D_{2}, D_{3}, D_{4}, D_{5}\right)>0$ and $g\left(u_{1}, u_{2}, u_{3}, u_{4}, u_{5}\right)=$ $c_{1}\left(u_{1}^{4}+u_{2}^{2}+u_{3}^{2}+u_{4}^{2}+u_{5}^{4}\right)$. Thus, $L \geq g\left(u_{1}, u_{2}, u_{3}, u_{4}, u_{5}\right)$ holds true and $g$ is continuous. Also, $g$ satisfies the condition $\left|\left(u_{1}, u_{2}, u_{3}, u_{4}, u_{5}\right)\right|^{-1} g\left(u_{1}, u_{2}, u_{3}, u_{4}, u_{5}\right) \rightarrow \infty$ whenever $\left|\left(u_{1}, u_{2}, u_{3}, u_{4}, u_{5}\right)\right| \rightarrow \infty$. Thus, all the conditions for the existence of controls are fulfilled (for more details one cam follow [28, 29]). Hence the result.

Now, we shall use Pontryagin's maximum principle for necessary conditions for optimal controls. For that the associated Hamiltonian $\mathcal{H}$ is given by

$$
\begin{aligned}
\mathcal{H}= & L\left(S, A, E, I_{1}, I_{2}, Q, R, Z, S_{v}, I_{v}, u_{1}, u_{2}, u_{3}, u_{4}, u_{5}\right)+\lambda_{1} \frac{d S}{d t}+\lambda_{2} \frac{d A}{d t}+\lambda_{3} \frac{d E}{d t} \\
& +\lambda_{4} \frac{d I_{1}}{d t}+\lambda_{5} \frac{d I_{2}}{d t}+\lambda_{6} \frac{d Q}{d t}+\lambda_{7} \frac{d R}{d t}+\lambda_{8} \frac{d Z}{d t}+\lambda_{9} \frac{d S_{v}}{d t}+\lambda_{10} \frac{d I_{v}}{d t}
\end{aligned}
$$

where $\lambda_{i}, 1 \leq i \leq 10$, are the adjoint variables. The following result characterizes the optimal controls.

Theorem 5.2. Let $u_{i}^{*}, 1 \leq i \leq 5$, be optimal control functions and $S^{*}, A^{*}, E^{*}, I_{1}^{*}, I_{2}^{*}, Q^{*}$, $R^{*}, Z^{*}, S_{v}^{*}, I_{v}^{*}$ are the corresponding state variables of the optimal control problem (2)-(3). Then there exists adjoint variable $\lambda=\left(\lambda_{1}, \lambda_{2}, \ldots, \lambda_{10}\right)^{T} \in \mathbb{R}^{10}$, which satisfies the following 
equations:

$$
\begin{aligned}
& \frac{d \lambda_{1}}{d t}=\lambda_{1} \mu+\left(\eta+\theta u_{1}\right) Z\left(\lambda_{1}-\lambda_{2}\right)+\beta_{2} A\left(\frac{I_{v}}{N^{2}}\right)\left(\lambda_{3}-\lambda_{2}\right) \\
& +\left(1-u_{2}\right) \beta_{1} I_{v}\left(\frac{A+E+I_{1}+I_{2}+Q+R}{N^{2}}\right)\left(\lambda_{1}-\lambda_{3}\right) \\
& +\left(1-u_{2}\right) \beta_{3} S_{v}\left(\frac{I_{1}+I_{2}}{N^{2}}\right)\left(\lambda_{10}-\lambda_{9}\right) \\
& \frac{d \lambda_{2}}{d t}=C_{4}+\lambda_{2} \mu+\gamma_{2}\left(\lambda_{2}-\lambda_{1}\right)+\beta_{2} I_{v}\left(\frac{S+E+I_{1}+I_{2}+Q+R}{N^{2}}\right)\left(\lambda_{2}-\lambda_{3}\right) \\
& +\left(1-u_{2}\right) \beta_{3} S_{v}\left(\frac{I_{1}+I_{2}}{N^{2}}\right)\left(\lambda_{10}-\lambda_{9}\right)+\left(1-u_{2}\right) \beta_{1} I_{v}\left(\frac{S}{N^{2}}\right)\left(\lambda_{3}-\lambda_{1}\right) \\
& \frac{d \lambda_{3}}{d t}=-C_{1}+\lambda_{3} \mu+m \alpha E\left(\lambda_{3}-\lambda_{5}\right)+\left(1-u_{2}\right) \beta_{1} I_{v}\left(\frac{S}{N^{2}}\right)\left(\lambda_{3}-\lambda_{1}\right) \\
& +\beta_{2} A\left(\frac{I_{v}}{N^{2}}\right)\left(\lambda_{3}-\lambda_{2}\right)+\left(1-u_{2}\right) \beta_{3} S_{v}\left(\frac{I_{1}+I_{2}}{N^{2}}\right)\left(\lambda_{10}-\lambda_{9}\right) \\
& \frac{d \lambda_{4}}{d t}=-C_{2}-a \lambda_{8}+\left(\mu+\mu_{1}\right) \lambda_{4}+\left(\gamma_{1}+\eta_{1} u_{3}\right)\left(\lambda_{4}-\lambda_{6}\right)+\left(1-u_{2}\right) \beta_{1} I_{v}\left(\frac{S}{N^{2}}\right)\left(\lambda_{3}-\lambda_{1}\right) \\
& +\beta_{2} A\left(\frac{I_{v}}{N^{2}}\right)\left(\lambda_{3}-\lambda_{2}\right)+\left(1-u_{2}\right) \beta_{2} S_{v}\left(\frac{S+E+A+Q+R}{N^{2}}\right)\left(\lambda_{9}-\lambda_{10}\right) \\
& \frac{d \lambda_{5}}{d t}=-C_{3}+\left(\mu+\mu_{2}\right) \lambda_{5}+\gamma\left(\lambda_{5}-\lambda_{8}\right)+\left(\delta+\eta_{2} u_{4}\right)\left(\lambda_{5}-\lambda_{4}\right)+\left(1-u_{2}\right) \beta_{1} I_{v}\left(\frac{S}{N^{2}}\right)\left(\lambda_{3}-\lambda_{1}\right) \\
& +\beta_{2} A\left(\frac{I_{v}}{N^{2}}\right)\left(\lambda_{3}-\lambda_{2}\right)+\left(1-u_{2}\right) \beta_{3} S_{v}\left(\frac{S+E+A+Q+R}{N^{2}}\right)\left(\lambda_{9}-\lambda_{10}\right) \\
& \frac{d \lambda_{6}}{d t}=\left(\mu+\mu_{3}\right) \lambda_{6}+\alpha_{1}\left(\lambda_{6}-\lambda_{7}\right)+\left(1-u_{2}\right) \beta_{1} I_{v}\left(\frac{S}{N^{2}}\right)\left(\lambda_{3}-\lambda_{1}\right) \\
& +\beta_{2} A\left(\frac{I_{v}}{N^{2}}\right)\left(\lambda_{3}-\lambda_{2}\right)+\left(1-u_{2}\right) \beta_{3} S_{v}\left(\frac{I_{1}+I_{2}}{N^{2}}\right)\left(\lambda_{10}-\lambda_{9}\right) \\
& \frac{d \lambda_{7}}{d t}=\mu \lambda_{7}+\left(1-u_{2}\right) \beta_{1} I_{v}\left(\frac{S}{N^{2}}\right)\left(\lambda_{3}-\lambda_{1}\right) \\
& +\beta_{2} A\left(\frac{I_{v}}{N^{2}}\right)\left(\lambda_{3}-\lambda_{2}\right)+\left(1-u_{2}\right) \beta_{3} S_{v}\left(\frac{I_{1}+I_{2}}{N^{2}}\right)\left(\lambda_{10}-\lambda_{9}\right) \\
& \frac{d \lambda_{8}}{d t}=a_{0} \lambda_{8}+\left(\eta+\theta u_{1}\right) S\left(\lambda_{1}-\lambda_{2}\right) \\
& \frac{d \lambda_{9}}{d t}=-C_{5}+\left(\mu_{v}+\eta_{3} u_{5}\right) \lambda_{9}+\left(1-u_{2}\right) \beta_{3}\left(\frac{I_{1}+I_{2}}{N}\right)\left(\lambda_{9}-\lambda_{10}\right) \\
& \frac{d \lambda_{10}}{d t}=-C_{5}+\left(\mu_{v}+\eta_{3} u_{5}\right) \lambda_{10}+\left(1-u_{2}\right) \beta_{1}\left(\frac{S}{N}\right)\left(\lambda_{1}-\lambda_{3}\right)+\beta_{2}\left(\frac{A}{N}\right)\left(\lambda_{2}-\lambda_{3}\right)
\end{aligned}
$$

with transversality conditions

$$
\lambda_{i}(T)=0,1 \leq i \leq 10, .
$$

Proof. Let $u_{i}^{*}, 1 \leq i \leq 5$, be the optimal control functions and $S^{*}, A^{*}, E^{*}, I_{1}^{*}, I_{2}^{*}, Q^{*}, R^{*}, Z^{*}$, 
$S_{v}^{*}, I_{v}^{*}$ are the corresponding state variables. Then, Pontryagin's Maximum Principle ensures the existence of the following adjoint variable $\lambda=\left(\lambda_{1}, \lambda_{2}, \ldots, \lambda_{10}\right)^{T} \in \mathbb{R}^{10}$, which satisfies the following canonical equations:

$$
\begin{gathered}
\frac{d \lambda_{1}}{d t}=-\frac{\partial H}{\partial S}, \quad \frac{d \lambda_{2}}{d t}=-\frac{\partial H}{\partial A}, \quad \frac{d \lambda_{3}}{d t}=-\frac{\partial H}{\partial E}, \quad \frac{d \lambda_{4}}{d t}=-\frac{\partial H}{\partial I_{1}}, \quad \frac{d \lambda_{5}}{d t}=-\frac{\partial H}{\partial I_{2}} \\
\frac{d \lambda_{6}}{d t}=-\frac{\partial H}{\partial Q}, \quad \frac{d \lambda_{7}}{d t}=-\frac{\partial H}{\partial R}, \quad \frac{d \lambda_{8}}{d t}=-\frac{\partial H}{\partial Z}, \quad \frac{d \lambda_{9}}{d t}=-\frac{\partial H}{\partial S_{v}} \text { and } \frac{d \lambda_{10}}{d t}=-\frac{\partial H}{\partial I_{v}} .
\end{gathered}
$$

with transversality conditions (6). Where $\mathcal{H}$ is the Hamiltonian defined as above. Thus, the adjoint system (5) can be obtained.

In the following result, we shall state the analytical forms of the optimal controls.

Theorem 5.3. The optimal controls $\left(u_{1}{ }^{*}, u_{2}{ }^{*}, u_{3}{ }^{*}, u_{4}{ }^{*}, u_{5}{ }^{*}\right)$ which minimizes $J$ over the region $U$ are given by

$$
\begin{aligned}
& u_{1}{ }^{*}=\min \left\{1, \max \left(0, \widetilde{u_{1}}\right)\right\} \\
& u_{2}{ }^{*}=\min \left\{1, \max \left(0, \widetilde{u_{2}}\right)\right\}, \\
& u_{3}{ }^{*}=\min \left\{1, \max \left(0, \widetilde{u_{3}}\right)\right\} \\
& u_{4}{ }^{*}=\min \left\{1, \max \left(0, \widetilde{u_{4}}\right)\right\}, \\
& u_{5}{ }^{*}=\min \left\{1, \max \left(0, \widetilde{u_{5}}\right)\right\},
\end{aligned}
$$

where

$$
\begin{gathered}
\widetilde{u_{1}}=\left(\frac{\theta Z S\left(\lambda_{1}-\lambda_{2}\right)}{4 D_{1}}\right)^{1 / 3}, \quad \widetilde{u_{2}}=\frac{\beta_{3} S_{v} \frac{I_{1}+I_{2}}{N}\left(\lambda_{10}-\lambda_{9}\right)+\beta_{1} S_{v} \frac{I_{v}}{N}\left(\lambda_{3}-\lambda_{1}\right)}{2 D_{2}}, \\
\widetilde{u_{3}}=\frac{\left(\lambda_{4}-\lambda_{5}\right) \eta_{1} I_{1}}{2 D_{3}}, \widetilde{u_{4}}=\frac{\left(\lambda_{5}-\lambda_{4}\right) \eta_{2} I_{2}}{2 D_{4}}, \widetilde{u_{5}}=\left(\frac{\lambda_{9} \eta_{3} S_{v}+\lambda_{10} \eta_{3} I_{v}}{4 D_{5}}\right)^{1 / 3} .
\end{gathered}
$$

Proof. : Using optimally condition, we have

$$
\frac{\partial \mathcal{H}}{\partial u_{1}}=0, \quad \frac{\partial \mathcal{H}}{\partial u_{2}}=0, \quad \frac{\partial \mathcal{H}}{\partial u_{3}}=0, \quad \frac{\partial \mathcal{H}}{\partial u_{4}}=0, \text { and } \frac{\partial \mathcal{H}}{\partial u_{5}}=0
$$

We have

$$
\frac{\partial H}{\partial u_{1}}=4 D_{1} u_{1}^{3}-\theta Z S \lambda_{1}+\theta Z S \lambda_{2}=0
$$

This gives

$$
u_{1}=\left(\frac{\theta Z S\left(\lambda_{1}-\lambda_{2}\right)}{4 D_{1}}\right)^{1 / 3}:=\widetilde{u_{1}} .
$$


Similarily

$$
\begin{gathered}
\frac{\partial H}{\partial u_{2}}=2 D_{2} u_{2}+\beta_{1} S \frac{I_{v}}{N} \lambda_{1}-\beta_{1} S \frac{I_{v}}{N} \lambda_{3}+\beta_{3} S_{v} \frac{I_{1}+I_{2}}{N} \lambda_{9}-\beta_{3} S_{v} \frac{I_{1}+I_{2}}{N} \lambda_{10}=0 \\
u_{2}=\frac{\beta_{3} S_{v} \frac{I_{1}+I_{2}}{N}\left(\lambda_{10}-\lambda_{9}\right)+\beta_{1} S_{v} \frac{I_{v}}{N}\left(\lambda_{3}-\lambda_{1}\right)}{2 D_{2}}:=\widetilde{u_{2}} . \\
\frac{\partial H}{\partial u_{3}}=2 D_{3} u_{3}-\lambda_{4} \eta_{1} I_{1}+\lambda_{5} \eta_{1} I_{1}=0 \\
u_{3}=\frac{\left(\lambda_{4}-\lambda_{5}\right) \eta_{1} I_{1}}{2 D_{3}}:=\widetilde{u_{3}} . \\
\frac{\partial H}{\partial u_{4}}=2 D_{4} u_{4}+\lambda_{4} \eta_{2} I_{2}-\lambda_{5} \eta_{2} I_{2}=0 \\
\frac{\partial H}{\partial u_{5}}=4 D_{5} u_{5}^{3}-\lambda_{9} \eta_{3} S_{v}-\lambda_{10} \eta_{3} I_{v}=0 \\
u_{5}=\left(\frac{\lambda_{9} \eta_{3} S_{v}+\lambda_{10} \eta_{3} I_{v}}{4 D_{5}}\right)^{1 / 3}:=\widetilde{u_{5}}
\end{gathered}
$$

Moreover, lower and upper bounds of these control are 0 and 1 respectively. Thus, if $\widetilde{u_{1}}>1$, $\widetilde{u_{2}}>1, \widetilde{u_{3}}>1, \widetilde{u_{4}}>1$, and $\widetilde{u_{5}}>1$, then

$$
u_{1}=u_{2}=u_{3}=u_{4}=u_{5}=1 .
$$

Also, if $\widetilde{u}_{1}<0, \widetilde{u}_{2}<0, \widetilde{u}_{3}<0, \widetilde{u}_{4}<0, \widetilde{u}_{5}<0$, then

$$
u_{1}=u_{2}=u_{3}=u_{4}=u_{5}=0 .
$$

Otherwise, we have

$$
u_{1}=\widetilde{u_{1}}, \quad u_{2}=\widetilde{u_{2}}, \quad u_{3}=\widetilde{u_{3}}, u_{4}=\widetilde{u_{4}} \text { and } u_{5}=\widetilde{u_{5}}
$$

Hence, for these controls $u_{1}{ }^{*}, u_{2}{ }^{*}, u_{3}^{*}, u_{4}^{*}, u_{5}^{*}$ we get optimum value of the function $J$.

\section{Numerical experimentations and discussion}

This part devotes to perform the numerical simulations for the control problem to further explore the effect of control interventions on the dynamics of the disease transmission. For this purpose, we shall define the following control policies:

Policy A: Execution of $u_{1}(t), u_{2}(t)$ and $u_{3}(t)$,

Policy B: Execution of $u_{1}(t), u_{3}(t)$ and $u_{5}(t)$,

Policy C: Execution of $u_{2}(t), u_{3}(t)$ and $u_{5}(t)$, 
Policy D: Execution of all controls $u_{1}(t)-u_{5}(t)$,

Policy E: Execution of $u_{1}(t), u_{3}(t)$ and $u_{4}(t)$,

Policy F: Implementation of only $u_{2}(t)$ and

Policy G: Implementation of only $u_{5}(t)$.

In order to compile the numerical simulations, a set of representative parameters is chosen as given below

$$
\begin{gathered}
\Lambda=20, \Lambda_{v}=1000, \beta_{1}=0.375, \beta_{2}=0.2, \beta_{3}=0.75, \mu=0.000042, \mu_{1}=0.0004 \\
\mu_{2}=0.004, \gamma=0.143, \gamma_{1}=0.01, \gamma_{2}=0.02, \delta=0.01, \alpha=0.1, \alpha_{1}=0.143, \mu_{v}=0.042 \\
\theta=0.01, \eta_{1}=0.1, \eta_{2}=0.1, \eta_{3}=0.1, \mu_{3}=0.004, \eta=0.1, a=0.1, a_{0}=0.05, m=0.2
\end{gathered}
$$

The time period for controls to be applied is $T=120$ days along with the initial population size $S(0)=10000, A(0)=10, E(0)=20, I_{1}(0)=5, I_{2}(0)=5, Q(0)=1, R(0)=1, Z(0)=1$, $S_{v}(0)=100000, I_{v}(0)=200$. We choose the positive weight constants as: $C_{1}=1.64, C_{2}=$ $1, C_{3}=1, C_{4}=0.1, C_{5}=1, D_{1}=10, D_{2}=10, D_{3}=10, D_{4}=10$, and $D_{5}=10$. Further, we simulate the optimality system (7) to explore the impact of applied controls numerically. A standard forward-backward sweep scheme is accounted for simulation of the optimality system (8). In this method, the optimal state system is solved starting forward in time using an initial guess for the optimal controls and after that the adjoint state system is solved backward in time using ode45 in MATLAB. Further, using these state and adjoint variables, the corresponding optimal controls are updated and continued this iterative process till a pre-defined convergence criterion is met (for details see [39]). The corresponding numerical outcomes for different control policies are depicted in Figures 5-12.

The counts/profiles of the both infected population (detected and undetected) for each control policy are plotted in Figure 5. One can easily see that Policy D (black colored curve) which includes all controls is found highly effective to reduce the cumulative count of the infected population. In addition, Policy A and Policy C (have similar impact) also play an important role in reducing the cumulative count of the infected population. Thus our finding suggests that use of bed nets and detection/quarantine along with either effect of information (Policy A) or effect of insecticides keep a strong tab on the cumulative count of the infection and have the equivalent effect. Therefore, in the absence of the information impact, we can implement the use of insecticides to minimise the burden of infection count within population and vice-versa. Furthermore, Policy B is found more effective than the Policy E in suppressing the epidemic peak during the course. Whereas, Policy E has strong impact on the detected infective population than the undetected one (curves given in magenta color in Figure 5). Thus, we infer that the comprehensive usage of bed nets and effect of information is more effective along with mosquito insecticides than the screening intervention of undetected human infective. Moreover, in between the single control strategies of only use of bed nets/mosquito repellents (Policy F) and mosquito insecticides (Policy G), the use of bed nets/mosquito repellents has remarkable capacity to suppress the epidemic burden (curves given in cyan color in Figure 5).

A similar effect of designed control policies as discussed above are also found in other human population as seen in Figure 6 and Figure 7. For example, the count of the exposed 

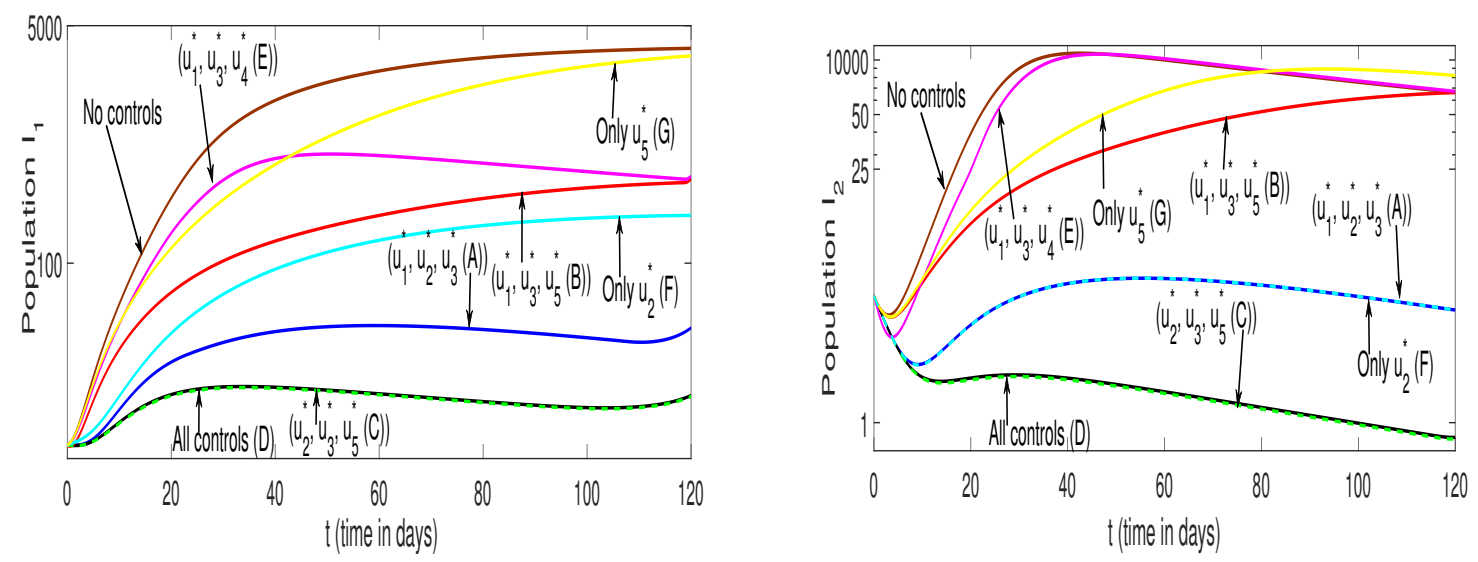

Figure 5: Profiles of $I_{1}$ and $I_{2}$.

population is at a very minimum level for control policies A, C and D (Figure 6(a)). In addition, the use of only bed nets/mosquito repellent (Policy F, cyan colored curve) plays a vital role in suppressing the count of exposed population than the policies B, E and G. Whereas, in the absence of the Policy F, the execution of the Policy B (in between policies $\mathrm{B}$ and E) may be a viable option for controlling the future infection (red colored curve in Figure 6(a)). Profiles of other human populations such as aware, quarantined and recovered are shown in Figure 6(b) and Figure 7. One can easily observe from Figure 6(b) that the count of the aware population settles at its maximum level during the course of epidemic when all the controls (Policy D) are implemented.
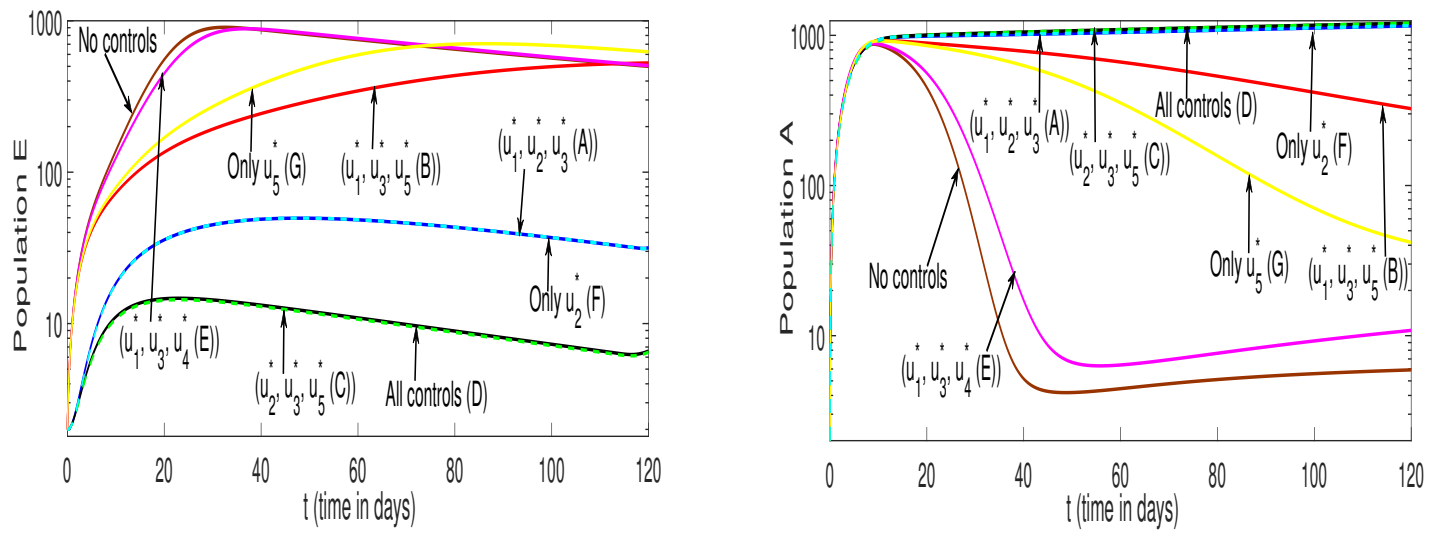

Figure 6: Profiles of $E$ and $A$.

The profiles of the mosquito population $\left(S_{v}\right.$ and $\left.I_{v}\right)$ are depicted for all control policies in Figure 8. We infer that the combined impact of all controls (Policy D) is highly effective in reducing the count of mosquito population. Whereas, the only usage of mosquito insecticides (Policy G) also has a strong impact on suppressing the count of the infected mosquito (yellow colored curve in Figure 8(a)). Moreover, in between the policies B and E, the policy makers can execute Policy B as it also has a significant impact on the mosquito population (red 

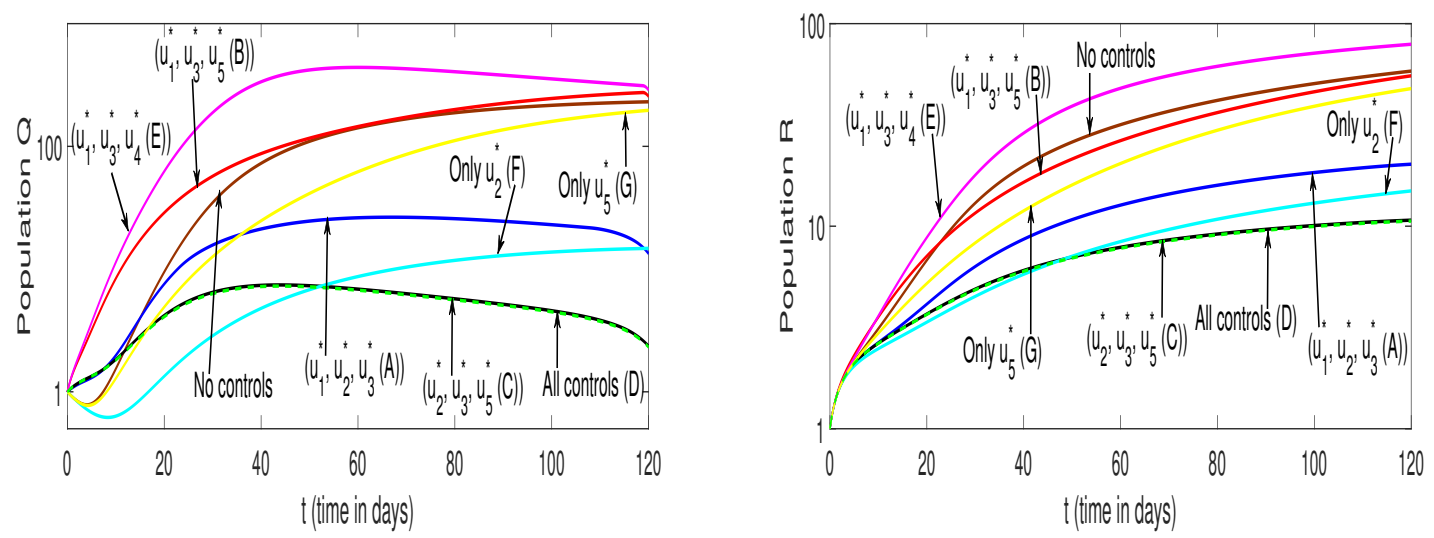

Figure 7: Profiles of $Q$ and $R$.

colored curve in Figure 8).
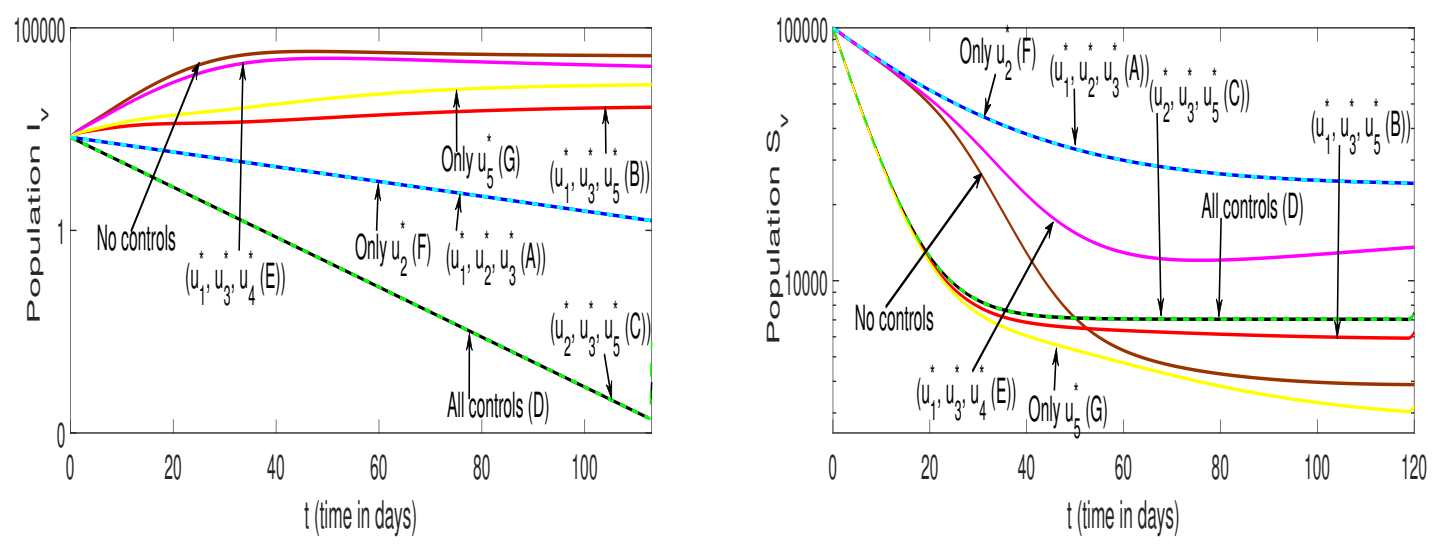

Figure 8: Profiles of $I_{v}$ and $S_{v}$.

The profiles of all optimal controls $\left(u_{1}^{*}-u_{5}^{*}\right)$ are plotted in Figures 9-11. Our findings infers that the optimal controls $u_{2}^{*}$ (Policy F) and $u_{5}^{*}$ (Policy G) are highly effective and have to be applied with full potential during the entire course of the epidemic to minimise the cumulative count of the human as well as mosquito infected population Figure $9(\mathrm{~b})$ and Figure 11. Whereas, optimal controls $u_{1}^{*}$ and $u_{3}^{*}$ have to be executed with various potential for different control policies as shown in Figure 9(a) and Figure 10(a). For instance, the optimal control $u_{3}^{*}$ has to be applied with fill potential in Policy E (curve in magenta) whereas in policies $\mathrm{C}$ and $\mathrm{D}$, a reduced potential (up to 60-70\%) is needed in controlling the disease spread (green and black colored curves). A similar impact for the optimal control $u_{4}^{*}$ is also observed as given in Figure 10(b). In the case of Policy D, a minimum potential is required than in the Policy E (black and magenta colored curves). 

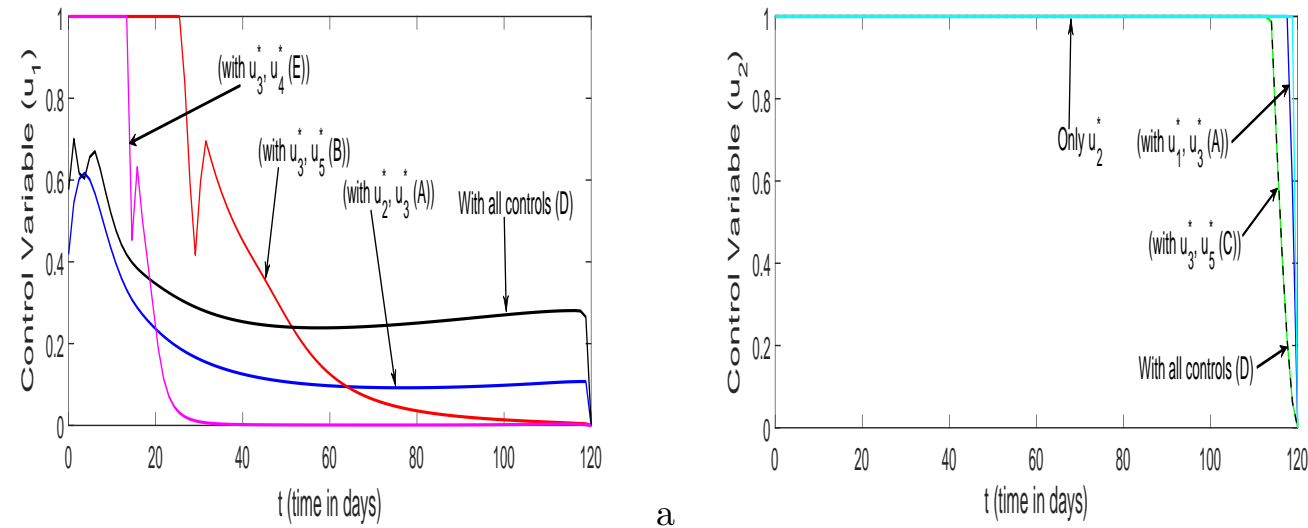

$\mathrm{b}$

Figure 9: Profiles of optimal controls $u_{1}^{*}$ and $u_{2}^{*}$.
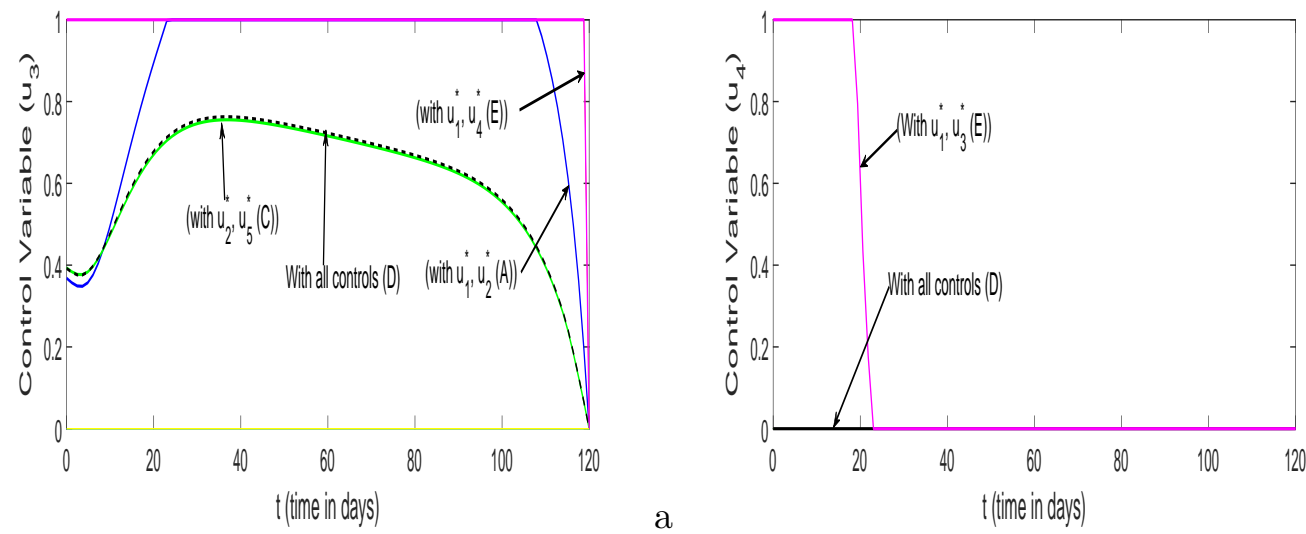

Figure 10: Profiles of optimal controls $u_{3}^{*}$ and $u_{4}^{*}$.

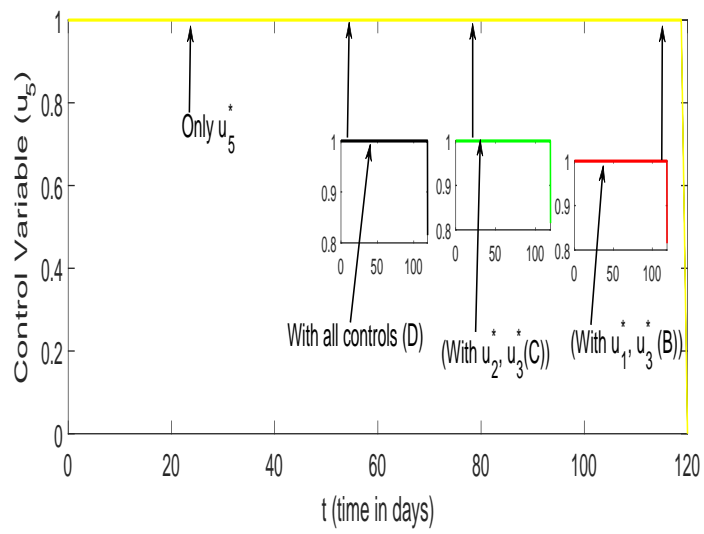

Figure 11: Profile of optimal control $u_{5}^{*}$. 


\subsection{Analysis of cost-effectiveness of control policies}

Execution of control interventions requires a large amount of the money which primarily includes the costs of medicines, diagnostic charges, hospitalization charges and the cost of man power needed. Thus, it becomes important to vet the cost-effectiveness, criticality and applicability of the applied control policies. Therefore, in this part, we shall discuss the cost effectiveness of applied control policies A-G by making a comparative study. The profiles of the cost distribution for each control policies are plotted in Figure 12. The Policy D is highly economically viable in controlling the disease spread. Moreover, policies A and $\mathrm{C}$ are also found significantly cost effective in reducing the disease burden (blue and green colored curves in Figure 12). Whereas, in between the policies F and G, Policy G (only $u_{5}^{*}$ ) is more cost effective than the Policy $\mathrm{F}$ (only $u_{2}^{*}$ ) while they have reverse impact on suppressing the count of the infected population either human or mosquito (cyan and yellow colored curves in Figure 12). Thus, we accentuate that the policy makers or health officials can select and execute the control policies according the present financial situation weather we need to suppress the infection with larger cost and vice-versa. Moreover, Policy E does not found much economically viable though it reduces the count of the detected infective human population and one can compare the magenta colored curves given in Figure 5)(a) and Figure 12. Therefore, some designed control policies may be less cost effective than others but have a huge impact on infection and vice-versa. Thus, policy makers have to very selective in implementation of suitable control policies.

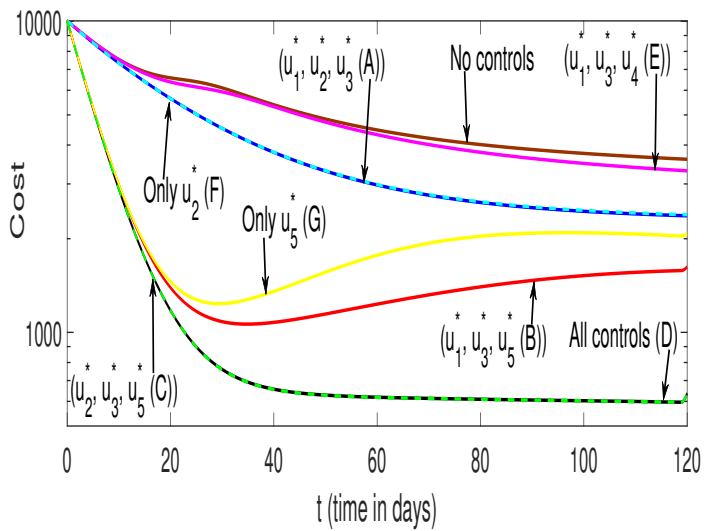

Figure 12: Profiles of Cost under various control policies (A-G).

\subsection{Impact of the basic reproduction number $R_{0}$ on control policies}

Generally, the basic reproduction number $R_{0}$ is an important factor that determines the severity of the disease persistence characterized by high epidemic peaks for its higher values [38]. Further, these epidemic peaks can be measured by different values of $R_{0}$ for various degree of transmissibility. Therefore, in the following, we shall made a comparative study for various values of $R_{0}$ by varying the degree of transmissibility on the optimal control policies (A-G) as well as on human and mosquito population. The basic reproduction number $R_{0}$ of 
the system (as obtained in 1 ), in the absence of all controls $u_{1}=0$ to $u_{5}=0$, is defined as:

$$
R_{0}=\sqrt{\frac{\beta_{1}}{\mu_{v}} a_{41}} .
$$

For all control policies, the high epidemic peaks of populations for a certain range of $R_{0}$ $\left(1 \leq R_{0} \leq 7\right)$ are plotted in Figures $13-16$. The maximum cost, in this case, is also depicted in Figure 17. One can easily found from Figure 13 that the policies A, C, and D are highly effective as the high epidemic peaks (high severity) are at a very low level for entire range of $R_{0}$. In between the policies $\mathrm{B}$ and $\mathrm{F}$, Policy $\mathrm{B}$ is more effective when epidemic is less severe $\left(1<R_{0}<4\right)$ than Policy $\mathrm{F}$ whereas, for high severity $\left(R_{0}>4\right)$, the Policy $\mathrm{F}$ is more constructive (red and cyan colored curves in Figure 13(a)). Moreover, Policy E and Policy G have similar capacity to reduce the epidemic peaks of detected infective while Policy $\mathrm{E}$ has less impact on undetected population as the peaks increase as $R_{0}$ increase (magenta colored curve in Figure 13(b)).
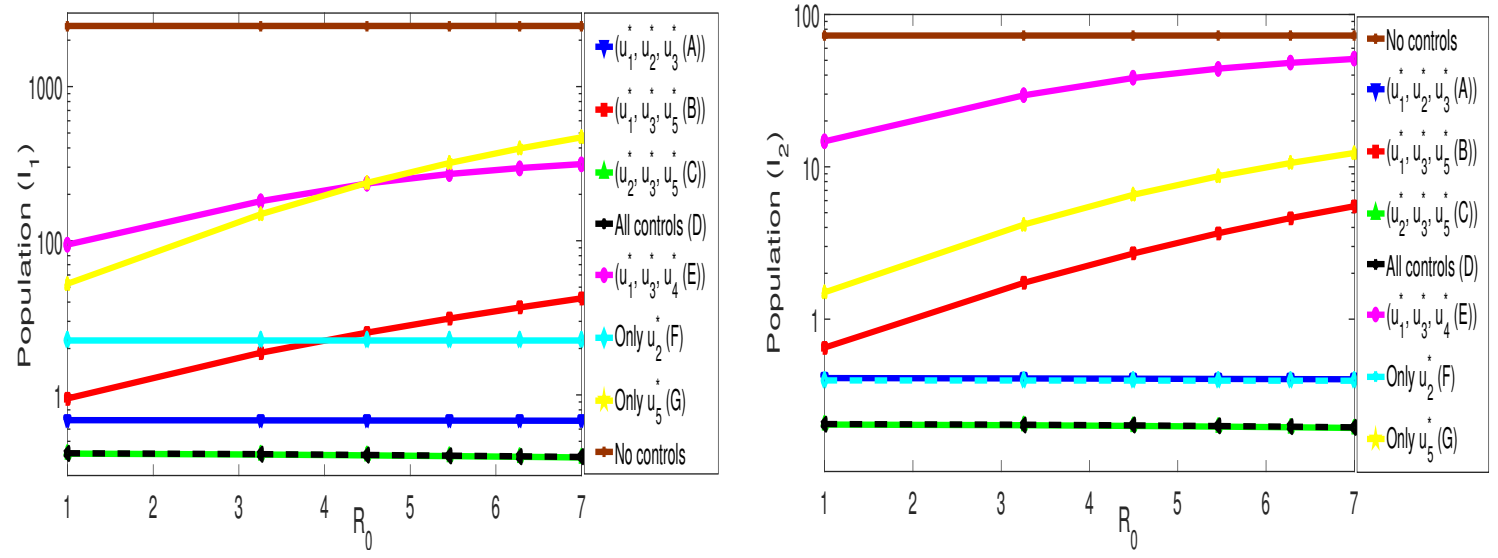

Figure 13: Profiles of $I_{1}$ and $I_{2}$ for various $R_{0}$.

Furthermore, the epidemic peaks for rest of the human populations are shown in Figures 14-15. A similar impact of all control policies are found in reducing the epidemic peaks for higher severity as well (the value of $R_{0}$ increases). Thus, the combined usage of all control interventions (Policy D) is found highly applicable in keeping the severity/epidemic peaks at a minimum level for entire range of $R_{0}$. Figure 16 corresponds to the peaks of mosquito populations for all values of $R_{0}$. Our study infers that the implementation of only mosquito insecticides (Policy G, $u_{5}^{*}$ ) plays an important in minimising the epidemic peaks for all most values of $R_{0}$ except the tail. Other policies such as $\mathrm{A}, \mathrm{C}$ and $\mathrm{D}$ also keep a tab on peaks of mosquito population while Policy E is less effective.

The corresponding maximum peaks of optimal cost for each control policies are demonstrated in Figure 17. As discussed in cost-effective part, here, we again found the similar impact. For instance, Policy F (only $u_{2}^{*}$ ) is found less cost-effective than Policy G (only $u_{5}^{*}$ ) for entire range of $R_{0}$ whereas it has great impact on infected population and vice-versa. Moreover, policies B, C and D are highly cost-effective even if epidemic is more severe. Policy E corresponds to less cost-effectiveness and it increases as $R_{0}$ increases. Thus, we conclude 

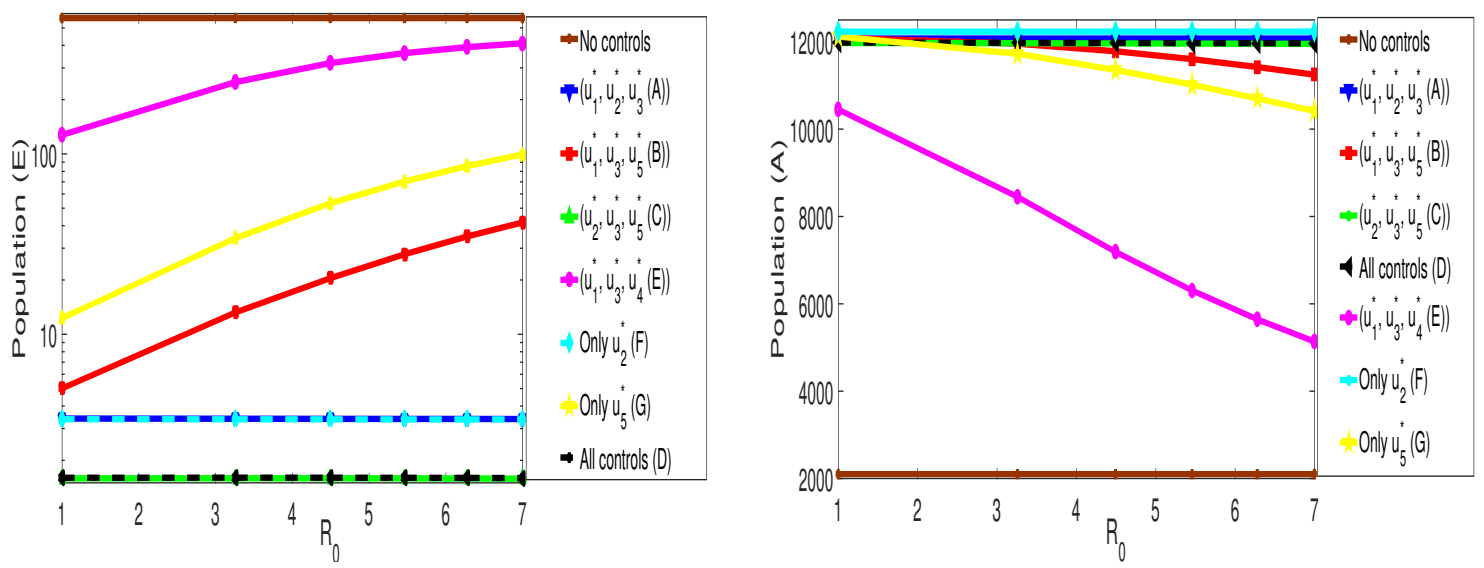

Figure 14: Profiles of $E$ and $A$ for various $R_{0}$.
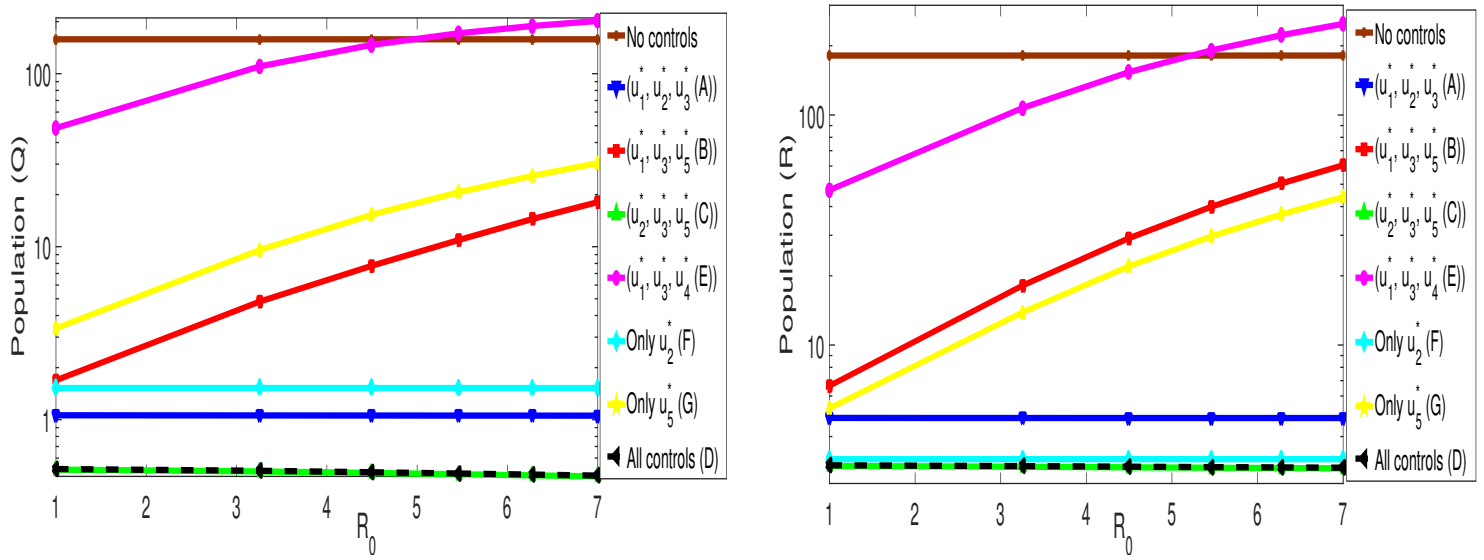

Figure 15: Profiles of $Q$ and $R$ for various $R_{0}$.
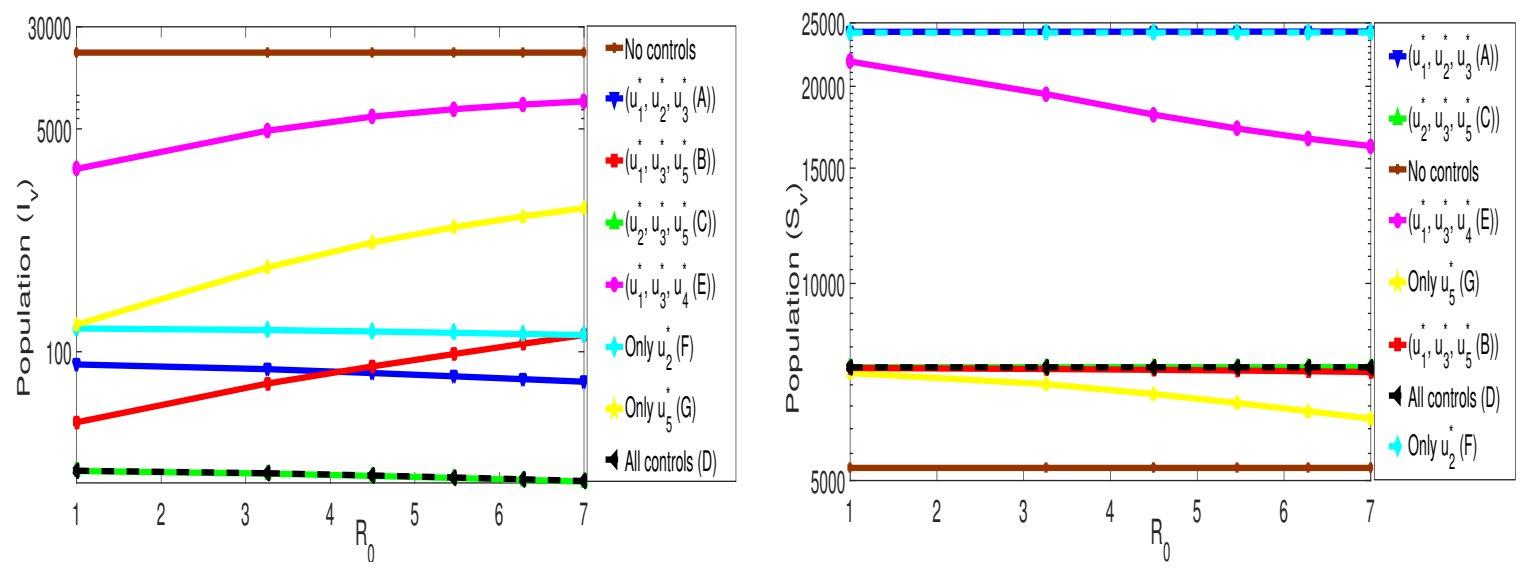

Figure 16: Profiles of $S_{v}$ and $I_{v}$ for various $R_{0}$. 
that the comprehensive impact of all controls (Policy D) is highly effective and economically viable in controlling the spread of disease within the population.

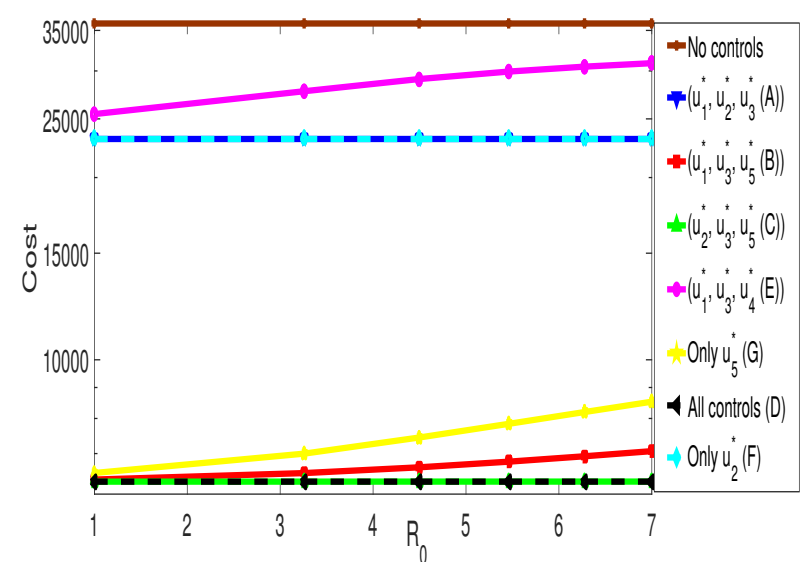

Figure 17: Profile of Cost for various $R_{0}$.

\section{Concluding Remarks}

In this work, a dengue model is formulated by quantifying the impact of information-induced behavioural change and separation of infected human class into two subparts - detected and undetected. In order to determine the key parameters of the model system, a sensitivity analysis is carried out that monitor the dynamics of dengue as well as the basic reproduction number $\left(\mathcal{R}_{0}\right)$. We found that the death rate of mosquito $\left(\mu_{v}\right)$ and the transmission rate between mosquito and human $\left(\beta_{1}\right)$ are crucial parameters which can help in reduction of $\mathcal{R}_{0}$. The parameters $\alpha, \gamma_{1} \delta, \mu_{v}$, and $\beta_{2}$ have also significant impact on the dynamics of dengue and particularly in infected human population.

We extended our proposed model to corresponding an optimal control problem by choosing five different controls measures such as: information induced behavioural change, use of mosquito repulsive, quarantine, screening and culling of mosquitoes. A cost functional that consists of various weighted costs is designed and characterization of optimal control paths analytically is performed with the help of Pontryagins Maximum Principle. In order to weigh the effect of various combinations of controls and provided the comparative study numerically, we proposed seven different control strategies, A to $\mathrm{G}$ (as mentioned in numerical section) by considering various combinations of above mentioned control measures. Our findings suggest that use of bed nets and detection/quarantine along with either effect of information (Strategy A) or effect of insecticides keep a strong tab on the cumulative count of the infection and have the equivalent effect. Similarly, other strategies also have significant on the prevalence of dengue. Whereas, the cumulative count of infected human population is found at a minimum level under the impact of Strategy D (all controls). Furthermore, we have also examined the effect of the basic reproduction number $\mathcal{R}_{0}$ on the designed control strategies (A to $\mathrm{G}$ ) and on dengue prevalence. For this purpose, we have estimated the high epidemic peaks subject to $\mathcal{R}_{0}$ for all strategies (A to G). Our study accentuates that the Strategies B, C and D are 
highly cost-effective even if epidemic is more severe (when the value of $\mathcal{R}_{0}$ is high). Whereas, Strategy E corresponds to less cost-effectiveness and it increases as $\mathcal{R}_{0}$ increases. Thus, we conclude that the comprehensive impact of all controls (Strategy D) is highly effective and economically viable in controlling the prevalence of dengue.

\section{Acknowledgement}

Mini Ghosh and Akhil Kumar Srivastav were supported by the research grants of DST, Govt. of India, via a sponsored research project: FILE NO. EMR/2017/003139. Prashant K Srivastava also acknowledge financial support from SERB (DST) project no MTR/2017/000803.

\section{Appendix A Optimality system}

The optimality system of the optimal control problem with minimized Hamiltonian $\mathcal{H}^{*}$ at $S=S^{*}, A=A^{*}, E=E^{*}, I_{1}=I_{1}^{*}, I_{2}=I_{2}^{*}, Q=Q^{*}, R=R^{*}, Z=Z^{*}, S_{v}=S_{v}^{*}, I_{v}=I_{v}^{*}$ and optimal controls $u_{i}=u_{i}^{*}$ for $i=1-5$ (Theorem 5.3) is given by

$$
\begin{aligned}
\frac{d S^{*}}{d t} & =\Lambda-\left(1-u_{2}^{*}(t)\right) \beta_{1} S^{*} \frac{I_{v}^{*}}{N^{*}}-\mu S^{*}-\left(\eta+\theta u_{1}^{*}(t)\right) Z^{*} S^{*}+\gamma_{2} A^{*} \\
\frac{d A^{*}}{d t} & =\left(\eta+\theta u_{1}^{*}(t)\right) Z^{*} S^{*}-\beta_{2} A^{*} \frac{I_{v}^{*}}{N^{*}}-\gamma_{2} A^{*}-\mu A^{*} \\
\frac{d E^{*}}{d t} & =\left(1-u_{2}^{*}(t)\right) \beta_{1} S^{*} \frac{I_{v}^{*}}{N^{*}}+\beta_{2} A^{*} \frac{I_{v}^{*}}{N^{*}}-\alpha E^{*}-\mu E^{*} \\
\frac{d I_{1}^{*}}{d t} & =(1-m) \alpha E^{*}-\left(\mu+\mu_{1}\right) I_{1}^{*}-\left(\gamma_{1}+\eta_{1} u_{3}^{*}(t)\right) I_{1}^{*}+\left(\delta+\eta_{2} u_{4}^{*}(t)\right) I_{2}^{*} \\
\frac{d I_{2}^{*}}{d t} & =m \alpha E^{*}-\left(\mu+\mu_{2}\right) I_{2}^{*}-\gamma I_{2}^{*}-\left(\delta+\eta_{2} u_{4}^{*}(t)\right) I_{2}^{*} \\
\frac{d Q^{*}}{d t} & =\left(\gamma_{1}+\eta_{1} u_{3}^{*}(t)\right) I_{1}^{*}-\left(\mu+\mu_{3}+\alpha_{1}\right) Q^{*} \\
\frac{d R^{*}}{d t} & =\alpha_{1} Q^{*}+\gamma I_{2}-\mu R^{*} \\
\frac{d Z^{*}}{d t} & =a I_{1}^{*}-a_{0} Z^{*}, \\
\frac{d S_{v}^{*}}{d t} & =\Lambda_{v}-\left(1-u_{2}^{*}(t)\right) \beta_{3} S_{v}^{*}\left(\frac{I_{1}^{*}+I_{2}^{*}}{N^{*}}\right)-\left(\mu_{v}+\eta_{3} u_{5}^{*}(t)\right) S_{v}^{*} \\
\frac{d I_{v}^{*}}{d t} & =\left(1-u_{2}^{*}(t)\right) \beta_{3} S_{v}^{*}\left(\frac{I_{1}^{*}+I_{2}^{*}}{N^{*}}\right)-\left(\mu_{v}+\eta_{3} u_{5}^{*}(t)\right) I_{v}^{*}
\end{aligned}
$$


The corresponding adjoint system at $\lambda_{i}=\lambda_{i}^{*}$ for $i=1-10$ is

$$
\begin{aligned}
& \frac{d \lambda_{1}}{d t}=\lambda_{1} \mu+\left(\eta+\theta u_{1}\right) Z\left(\lambda_{1}-\lambda_{2}\right)+\beta_{2} A\left(\frac{I_{v}}{N^{2}}\right)\left(\lambda_{3}-\lambda_{2}\right) \\
& +\left(1-u_{2}\right) \beta_{1} I_{v}\left(\frac{A+E+I_{1}+I_{2}+Q+R}{N^{2}}\right)\left(\lambda_{1}-\lambda_{3}\right) \\
& +\left(1-u_{2}\right) \beta_{3} S_{v}\left(\frac{I_{1}+I_{2}}{N^{2}}\right)\left(\lambda_{10}-\lambda_{9}\right) \\
& \frac{d \lambda_{2}}{d t}=C_{4}+\lambda_{2} \mu+\gamma_{2}\left(\lambda_{2}-\lambda_{1}\right)+\beta_{2} I_{v}\left(\frac{S+E+I_{1}+I_{2}+Q+R}{N^{2}}\right)\left(\lambda_{2}-\lambda_{3}\right) \\
& +\left(1-u_{2}\right) \beta_{3} S_{v}\left(\frac{I_{1}+I_{2}}{N^{2}}\right)\left(\lambda_{10}-\lambda_{9}\right)+\left(1-u_{2}\right) \beta_{1} I_{v}\left(\frac{S}{N^{2}}\right)\left(\lambda_{3}-\lambda_{1}\right) \\
& \frac{d \lambda_{3}}{d t}=-C_{1}+\lambda_{3} \mu+m \alpha E\left(\lambda_{3}-\lambda_{5}\right)+\left(1-u_{2}\right) \beta_{1} I_{v}\left(\frac{S}{N^{2}}\right)\left(\lambda_{3}-\lambda_{1}\right) \\
& +\beta_{2} A\left(\frac{I_{v}}{N^{2}}\right)\left(\lambda_{3}-\lambda_{2}\right)+\left(1-u_{2}\right) \beta_{3} S_{v}\left(\frac{I_{1}+I_{2}}{N^{2}}\right)\left(\lambda_{10}-\lambda_{9}\right) \\
& \frac{d \lambda_{4}}{d t}=-C_{2}-a \lambda_{8}+\left(\mu+\mu_{1}\right) \lambda_{4}+\left(\gamma_{1}+\eta_{1} u_{3}\right)\left(\lambda_{4}-\lambda_{6}\right)+\left(1-u_{2}\right) \beta_{1} I_{v}\left(\frac{S}{N^{2}}\right)\left(\lambda_{3}-\lambda_{1}\right) \\
& +\beta_{2} A\left(\frac{I_{v}}{N^{2}}\right)\left(\lambda_{3}-\lambda_{2}\right)+\left(1-u_{2}\right) \beta_{2} S_{v}\left(\frac{S+E+A+Q+R}{N^{2}}\right)\left(\lambda_{9}-\lambda_{10}\right) \\
& \frac{d \lambda_{5}}{d t}=-C_{3}+\left(\mu+\mu_{2}\right) \lambda_{5}+\gamma\left(\lambda_{5}-\lambda_{8}\right)+\left(\delta+\eta_{2} u_{4}\right)\left(\lambda_{5}-\lambda_{4}\right)+\left(1-u_{2}\right) \beta_{1} I_{v}\left(\frac{S}{N^{2}}\right)\left(\lambda_{3}-\lambda_{1}\right) \\
& +\beta_{2} A\left(\frac{I_{v}}{N^{2}}\right)\left(\lambda_{3}-\lambda_{2}\right)+\left(1-u_{2}\right) \beta_{3} S_{v}\left(\frac{S+E+A+Q+R}{N^{2}}\right)\left(\lambda_{9}-\lambda_{10}\right) \\
& \frac{d \lambda_{6}}{d t}=\left(\mu+\mu_{3}\right) \lambda_{6}+\alpha_{1}\left(\lambda_{6}-\lambda_{7}\right)+\left(1-u_{2}\right) \beta_{1} I_{v}\left(\frac{S}{N^{2}}\right)\left(\lambda_{3}-\lambda_{1}\right) \\
& +\beta_{2} A\left(\frac{I_{v}}{N^{2}}\right)\left(\lambda_{3}-\lambda_{2}\right)+\left(1-u_{2}\right) \beta_{3} S_{v}\left(\frac{I_{1}+I_{2}}{N^{2}}\right)\left(\lambda_{10}-\lambda_{9}\right) \\
& \frac{d \lambda_{7}}{d t}=\mu \lambda_{7}+\left(1-u_{2}\right) \beta_{1} I_{v}\left(\frac{S}{N^{2}}\right)\left(\lambda_{3}-\lambda_{1}\right) \\
& +\beta_{2} A\left(\frac{I_{v}}{N^{2}}\right)\left(\lambda_{3}-\lambda_{2}\right)+\left(1-u_{2}\right) \beta_{3} S_{v}\left(\frac{I_{1}+I_{2}}{N^{2}}\right)\left(\lambda_{10}-\lambda_{9}\right) \\
& \frac{d \lambda_{8}}{d t}=a_{0} \lambda_{8}+\left(\eta+\theta u_{1}\right) S\left(\lambda_{1}-\lambda_{2}\right) \\
& \frac{d \lambda_{9}}{d t}=-C_{5}+\left(\mu_{v}+\eta_{3} u_{5}\right) \lambda_{9}+\left(1-u_{2}\right) \beta_{3}\left(\frac{I_{1}+I_{2}}{N}\right)\left(\lambda_{9}-\lambda_{10}\right) \\
& \frac{d \lambda_{10}}{d t}=-C_{5}+\left(\mu_{v}+\eta_{3} u_{5}\right) \lambda_{10}+\left(1-u_{2}\right) \beta_{1}\left(\frac{S}{N}\right)\left(\lambda_{1}-\lambda_{3}\right)+\beta_{2}\left(\frac{A}{N}\right)\left(\lambda_{2}-\lambda_{3}\right)
\end{aligned}
$$

with transversality conditions (6). 


\section{References}

[1] M.G. Guzman, G. Kouri, Dengue: an update, Lancet Infectious Disease 2(1) (2002) 33-42.

[2] T.L. Bancroft, On the etiology of dengue fever, Australasian Medical Gazette 25 (1906) $17-18$.

[3] D. Gubler, Dengue and dengue hemorrhagic fever, Clinical Microbiology Review, 11(3) (1998) 480-496.

[4] World Health Organization. Dengue and severe dengue, (who2013). Available at http://www.who.int/mediacentre/factsheets/fs117/en/index.html.

[5] J. Whitehorn, J. Farrar, Dengue, British Medical Bulletin 95(1) (2010) 161-173.

[6] J.E. Blaney, J. Matro, B. Murphy, S. Whitehead, Recombinant, live attenuated tetravalent dengue virus vaccine formulations induce a balanced, broad, and protective neutralizing antibody response against each of the four serotypes in rhesus monkeys, Journal of Virology 79(9) (2005) 5516-5528.

[7] Center for Disease Control. Dengue fact sheet, (cdc2007). Available at http://www.cdc.gov/ncidod/dvbid/dengue/resources/DengueFactSheet.pdf.

[8] Center for Vaccine Development. Live attenuated tetravalent den vaccine, (cvd2007). http://www.denguevaccines.org/live-attenuated-vaccines.

[9] N. Gratz, Emergency control of Aedes aegypti as a disease vector in urban areas, Journal of the American Mosquito Control Association 7(3) (1991) 353-365.

[10] Dengue guidelines for diagnosis,treatment, prevention and control ,2009, https : //www.ncbi.nlm.nih.gov/books/NBK143157/pdf / Bookshel $f_{N}$ BK143157.pdf,France.

[11] https://nvbdcp.gov.in/index1.php?lang=1\&level=1\&sublinkid=5784\&lid=3689

[12] https://www.malariasite.com/malaria-india/

[13] E. Gupta, L. Dar, G. Kapoor, S. Broor, 2006. The changing epidemiology of dengue in Delhi. India. Virology Journal 3, 92.

[14] L. Esteva , C. Vargas, Analysis of a dengue disease transmission model. Mathematical Bioscience, 150 (2),(1998) 131-151.

[15] L. Esteva, C. Vargas, A model for dengue disease with variable human population,Mathematical Bioscience 38 (3),(1999) 220-240 .

[16] M. Amaku, F.A.B. Coutinho, S. M. Raimundo, L. F. Lopez, M.N.Burattini, E. Massad, A comparative analysis of the relative efficacy of vector-control strategies against dengue fever. Bulletin of Mathematical Biology, 76,(2013) 697-717 .

[17] A. K. Srivastav, M. Ghosh, Assessing the impact of treatment on the dynamics of dengue fever: A case study of India,Applied Mathematics and Computation, 362, (2019), 124533. 
[18] S. A. Carvalho, S.O. da Silva, I. da C. Charret,Mathematical modeling of dengue epidemic: control methods and vaccination strategies, Theory in Biosciences (2019),https://doi.org/10.1007/s12064-019-00273-7

[19] A. Abdelrazec, J. Belair, C. Shan, H. Zhu, Modeling the spread and control of dengue with limited public health resources. Mathematical Bioscience.271:136145. (2016) DOI: 10.1016/j. mbs.2015.11.004.

[20] A. K. Srivastav, P. K. Tiwari and M. Ghosh,Modeling the impact of early case detection on dengue transmission: deterministic vs. stochastic,Stochastic Analysis and Applications,(2020) https://doi.org/10.1080/07362994.2020.1804403

[21] I. Ghosh, P.K. Tiwari, J. Chattopadhyay, Effect of active case finding on dengue control: Implications from a mathematical model. Journal of Theoretical Biology 464:50-62(2019).

[22] T-T Zheng, L-F Nie, Modelling the transmission dynamics of two-strain Dengue in the presence awareness and vector control, Journal of Theoretical Biology, 443, (2018), 82-91

[23] K. Blayneh, Y. Cao, H.D. Kwon, Optimal control of vector-borne diseases: Treatment and prevention. Discrete \& Continuous Dynamical Systems - B, 2009, 11 (3) : 587-611.

[24] T Rawson, KE Wilkins, MB Bonsall, Optimal control approaches for combining medicines and mosquito control in tackling dengue. Royal Society Open Science, 7:181843 (2020)

[25] P Pongsumpun , I.-Ming Tang and N. Wongvanich,Optimal control of the dengue dynamical transmission with vertical transmission, Advances in Difference Equations (2019) 2019:176

[26] H S. Rodrigues. T. T.Monteiro, D. F.M.Torres, Vaccination models and optimal control strategies to dengue, Mathematical Biosciences, 247, (2014), 1-12.

[27] A. Fischer, K. Chudej, H. Josef Pesch,Optimal vaccination and control strategies against dengue, Mathematical methods in applied sciences, 42(10) , (2019),3496-3507.

[28] A. Kumar, P.K. Srivastava, Y. Dong, Y. Takeuchi, Optimal control of infectious disease: Information-induced vaccination and limited treatment, Physica A (2019), https://doi.org/10.1016/j.physa.2019.123196.

[29] A. Kumar, P. K.Srivastava, Vaccination and treatment as control interventions in an infectious disease model with their cost optimization, Communications in Nonlinear Science and Numerical Simulation, 44, (2017), 334-343.

[30] A dOnofrio, P Manfredi, and E Salinelli. Vaccinating behaviour, information, and the dynamics of SIR vaccine preventable diseases. Theoretical Population Biology, 71:301-317, 2007

[31] A. Kumar,P. K.Srivastava, , RP Gupta Nonlinear dynamics of infectious diseases via information-induced vaccination and saturated treatment, Mathematics and Computer in Simulation (2018), https://doi.org/10.1016/j.matcom.2018.09.024 
[32] P. van den Driessche, J. Watmough, Reproduction numbers and sub-threshold endemic equilibria for compartmental models of disease transmission, Mathematical Bioscience, 180 (2002), 29-48.

[33] S.M. Blower, H. Dowlatabadi, Sensitivity and uncertainty analysis of complex models of disease transmission: an HIV model, as an example, International Statistical Review, 62 (1994) 229-243.

[34] S. Marino, I.B. Hogue, C.J. Ray, D.E. Kirschner, A methodology for performing global uncertainty and sensitivity analysis in systems biology, Journal of Theoretical Biology 254(1) (2008)178-196.

[35] A. Chakravarti, R. Arora, C. Luxemburger, Fifty years of dengue in India, Transactions of the Royal Society of Tropical Medicine \& Hygiene and International Health 106 (2012) $273-282$.

[36] National Vector Borne Disease Control Programme. Dengue fact sheet, (nvbdcp2018). Available at http://www.nvbdcp.gov.in/DENGU1.html.

[37] World Health Organization (WHO), Health of older persons in the western pacific region; Country Profiles(1998).

[38] S Lee, G Chowell, and C Castillo-Chávez. Optimal control for pandemic influenza: the role of limited antiviral treatment and isolation. Journal of Theoretical Biology, $265(2): 136-150,2010$.

[39] SM Lenhart and JT Workman. Optimal control applied to biological models, 15. CRC Press, 2007. 